\title{
Constraining the population of cosmic ray protons in cooling flow clusters with $\gamma$-ray and radio observations: Are radio mini-halos of hadronic origin?
}

\begin{abstract}
C. Pfrommer and T. A. Enßlin
Max-Planck-Institut für Astrophysik, Karl-Schwarzschild-Str. 1, Postfach 1317, 85741 Garching, Germany

Received 18 April 2003 / Accepted 12 September 2003

Abstract. We wish to constrain the cosmic-ray proton (CRp) population in galaxy clusters. By hadronic interactions with the thermal gas of the intra-cluster medium (ICM), the CRp produce $\gamma$-rays for which we develop an analytic formalism to deduce their spectral distribution. Assuming the CRp-to-thermal energy density ratio $X_{\mathrm{CRp}}$ and the CRp spectral index to be spatially constant, we derive an analytic relation between the $\gamma$-ray and bolometric X-ray fluxes, $\mathcal{F}_{\gamma}$ and $F_{\mathrm{X}}$. Based on our relation, we compile a sample of suitable clusters which are promising candidates for future detection of $\gamma$-rays resulting from hadronic CRp interactions. Comparing to EGRET upper limits, we constrain the CRp population in the cooling flow clusters Perseus and Virgo to $X_{\mathrm{CRp}}<20 \%$. Assuming a plausible value for the CRp diffusion coefficient $\kappa$, we find the central CRp injection luminosity of M 87 to be limited to $10^{43} \mathrm{erg} \mathrm{s}^{-1} \kappa /\left(10^{29} \mathrm{~cm}^{2} \mathrm{~s}^{-1}\right)$. The synchrotron emission from secondary electrons generated in CRp hadronic interactions allows even tighter limits to be placed on the CRp population using radio observations. We obtain excellent agreement between the observed and theoretical radio brightness profiles for Perseus, but not for Coma without a radially increasing CRp-to-thermal energy density profile. Since the CRp and magnetic energy densities necessary to reproduce the observed radio flux are very plausible, we propose synchrotron emission from secondary electrons as an attractive explanation of the radio mini-halos found in cooling flow clusters. This model can be tested with future sensitive $\gamma$-ray observations of the accompanying $\pi^{0}$-decays. We identify Perseus (A 426), Virgo, Ophiuchus, and Coma (A 1656) as the most promising candidate clusters for such observations.
\end{abstract}

Key words. galaxies: cooling flows - galaxies: cluster: general - galaxies: cluster: individual: Perseus (A426) galaxies: intergalactic medium - ISM: cosmic rays - radiation mechanisms: non-thermal

\section{Introduction}

Cooling flows are regions where the influence of non-thermal intra-cluster medium (ICM) components such as magnetic fields and cosmic rays may be strongest within a galaxy cluster owing to strong observed magnetic fields, central active galaxies, and increasing non-thermal-to-thermal energy ratio due to rapid thermal cooling processes. They are also regions where such components are best detectable due to the high gas density which allows for secondary particle production in hadronic interactions of cosmic ray nuclei with the ambient gas. By the term cooling flow we do not rely on specific models but only on observed properties such as declining temperature gradients and enhanced electron density profiles towards the center of the cluster.

Non-thermal relativistic particle populations such as cosmic ray electrons (CRe) and protons (CRp) can be injected into the ICM mainly by three different processes (following

Send offprint requests to: $\mathrm{C}$. Pfrommer,

e-mail: pfrommer@mpa-garching.mpg.de
Brunetti 2002) which produce radio signatures that differ morphologically as well as spectrally:

1. Shock acceleration: Natural acceleration mechanisms providing relativistic particles are strong structure formation and merger shocks (e.g., Harris et al. 1980; Sarazin 1999). Detailed studies have been undertaken on shocks of cosmological scales (Miniati et al. 2000; Takizawa \& Naito 2000). Fermi I acceleration processes of CRe at these shock fronts produce large scale extended peripheral radio relics as proposed by Enßlin et al. (1998a). For instance two prominent relics in Abell 3667 (Röttgering et al. 1997) were successfully reproduced in a simulation of this process by Roettiger et al. (1999).

2. Reaccelerated electrons: Secondly, reacceleration processes of mildly relativistic $\operatorname{CRe}(\gamma \simeq 100-300)$ being injected over cosmological timescales into the ICM by sources like radio galaxies, supernova remnants, merger shocks, and galactic winds can provide an efficient supply of highly-energetic CRe. Owing to their long lifetimes of a few times $10^{9}$ years these mildly relativistic CRe can 
accumulate within the ICM (see Sarazin 2002, and references therein), until they experience continuous in-situ acceleration either via shock acceleration or resonant pitch angle scattering by turbulent Alvén waves as originally proposed by Jaffe (1977), reconsidered by Schlickeiser et al. (1987), and lately by Ohno et al. (2002). These acceleration processes of CRe possibly yield extended radio halos centered on the cluster (Brunetti et al. 2001) while there are also suggestions that radio mini-halos within a cooling flow cluster originate from these processes (Gitti et al. 2002). There is also evidence that reacceleration processes acting on fossil radio plasma produces small filamentary radio relics at the cluster periphery, so-called revived radio ghosts (Enßlin \& Gopal-Krishna 2001; Enßlin \& Brüggen 2002) presumably by adiabatic compression in shock waves.

3. Particles of hadronic origin: Eventually, CRp can interact hadronically with the thermal ambient gas producing secondary electrons, neutrinos, and $\gamma$-rays in inelastic collisions taking place throughout the cluster volume which would generate radio halos through synchrotron emission (first pointed out by Dennison 1980; Vestrand 1982). In the ICM the CRp have lifetimes of the order of the Hubble time (Völk et al. 1996; Enßlin et al. 1997; Berezinsky et al. 1997), long enough to diffuse away from the production site and to maintain a distribution over the cluster volume. This process was reconsidered in more detail by Blasi \& Colafrancesco (1999) and by Dolag \& Enßlin (2000), the latter authors using numerical hydro-dynamical simulations including magnetic fields. Recently, Miniati et al. (2001b) have performed cosmological simulations of cluster formation including injection processes of primary CRp. These authors conclude that under certain conditions extended diffuse radio emission could be due to hadronically produced CRe. However, there are also claims that extended radio halos cannot be generated by secondary electrons due to the morphological steepness of predicted radio brightness profiles in contrast to observations (Brunetti 2002). Besides constraining the population of CRp in the ICM, this work will present arguments for the hadronic origin of radio mini-halos or a substantial contribution of secondary electrons to these mini-halos. We further perform a parameter study which shows that the large cluster radio halos could be also of hadronic origin, provided the CRpto-thermal energy density profile is radially increasing.

It is very difficult to distinguish between contributions of these three populations of cosmic ray (CR) particles to non-thermal particle populations, especially if all of them account for injection of cosmic rays into the ICM in different strength depending on underlying governing physical processes and parameters. The hadronically produced CRe may be reaccelerated by shocks or cluster turbulence and therefore mix up the different CRe populations.

Radio observations of the radio halo in the Coma cluster find a strong steepening of the synchrotron spectrum with increasing radius (Giovannini et al. 1993). This behavior is expected for a reaccelerated population of CRe (Brunetti et al. 1999, 2001). There is also a report of radial spectral steepening in the case of the radio mini-halo of Perseus according to Sijbring (1993). This, however, could easily be an observational artifact owing to a poor signal-to-noise ratio in the outer core parts of the cluster in combination with the ambiguity of determining the large scale Fourier components owing to the nonuniform coverage of the Fourier plane and missing shortbaseline information: the so-called "missing zero spacing"problem of interferometric radio observations. By comparing the spectral index distribution of the three radio maps $(92 \mathrm{~cm}$, $49 \mathrm{~cm}$, and $21 \mathrm{~cm}$ ), there seems to be likewise a possibility of radial spectral flattening depending on the chosen radial direction. The hadronic electron model does not necessarily produce the radial spectral steepening without fine-tuning.

Assumptions: The purpose of this work is to provide conceptually simple analytic instruments for describing the spectral signatures in radio, X-rays, and $\gamma$-rays resulting from inelastic cosmic ray ion collisions. It is especially important to constrain the population of CRp within clusters of galaxies in order to understand the governing physical processes of these objects and the important theoretical implications for the nonthermal content of the ICM, i.e. if non-thermal CR pressure plays an important role in supporting the intra-cluster ionized gas (Enßlin et al. 1997). The assumptions of our models are:

- CRe are taken to originate from hadronic interactions of CRp with thermal ambient protons of the ICM and the CRp population is described by a power-law distribution in momentum. The origin of this population is not specified here, but CRp may be accelerated by shock waves of cluster mergers, accretion shocks (Colafrancesco \& Blasi 1998), or injected from radio galaxies into the ICM (Valtaoja 1984; Enßlin et al. 1997; Blasi \& Colafrancesco 1999), or result from supernova driven galactic winds (Völk et al. 1996).

- In our isobaric model, the energy density of CRp is assumed to be proportional to the thermal energy density of the ICM. In our scenario of adiabatic compression of CRp during the formation of the cooling flow this proportionality is imposed prior to the transition. This assumption is reasonable if the thermal electron population and the CRp were energized by the same shock wave assuming that there is a constant fraction of energy going into the CRp population by such an acceleration process. As a third model we take a single central point source injecting the CRp which results in a very peaked CRp profile (compare Blasi 1999; Blasi \& Colafrancesco 1999).

- The CRp spectral index is assumed to be independent of position and therefore constant over the cluster volume. In some sense this represents an oversimplification which could be abandoned in order to reproduce some specific observational results, which however would be questionable without understanding the underlying physical processes.

- The electron density and temperature profiles of the ICM are assumed to be spherically symmetric and were taken from the literature. This assumption is justified in the case of $\gamma$-rays resulting from neutral pion decay because we use cluster volume averaged spectra in order to compare to observation, and is not severe in the case of radio emission, since the profiles are obtained from deprojected X-ray data. 
The magnetic field configuration is assumed to be spherically symmetric on cluster core scales and follows the electron density with a power-law index as a free parameter within the suggested range (Dolag et al. 2001, 1999).

- No reacceleration or diffusion process of CRe is taken into account in calculating the synchrotron and the inverse Compton (IC) emission. Therefore we provide conservative estimates for the flux.

- The radio spectrum is taken to be quasi-stationary owing to the short electron cooling time which establishes a stationary CRe population on very short timescales. There is a one-to-one correspondence between the CRp power law index and that of the CRe population which is in addition determined by radiative synchrotron losses and IC cooling.

The paper is organized in two main parts: It starts with theoretic modeling of $\gamma$-ray spectra resulting from hadronic CRp-p interactions and presents models of synchrotron and IC radiation emitted by secondary electrons. The second part discusses the astrophysical application of this formalism to a nearby cluster sample including cooling flow clusters. After modeling the spatial distribution of CRp within cooling flow clusters we constrain this population by comparing to EGRET upper limits. We furthermore obtain limits on the CRp population by the morphology of radio brightness profiles in the case of Perseus and Coma. Throughout this paper we assume the standard $\Lambda \mathrm{CDM}$ cosmology with $\Omega_{\mathrm{M}}=0.3, \Omega_{\Lambda}=0.7$, and $H_{0}=70 h_{70} \mathrm{~km} \mathrm{~s}^{-1} \mathrm{Mpc}^{-1}$, where $h_{70}$ indicates the scaling with $H_{0}$.

\section{Theoretic modeling of multi-frequency signatures resulting from hadronic CRp interactions}

In order to study non-thermal emission from clusters we model the IC and synchrotron radiation of secondary CRe produced in inelastic collisions by CRp scattering off thermal nuclei as well as the $\gamma$-ray spectrum produced by decaying pions being produced by these CRp-p collisions. After introducing our definitions (Sect. 2.1), we develop an analytic formalism describing the decay of secondary neutral pions into two high-energy $\gamma$-rays (Sect. 2.2). Section 2.2.2 uses the analytical fireball model for inelastic CRp interactions with nuclei of the intergalactic medium in the high-energy regime of CRp $\left(E_{\mathrm{p}} \gg\right.$ $m_{\mathrm{p}} c^{2}$ ), following Mannheim \& Schlickeiser (1994). Based on that we develop in Sect. 2.2.3 an analytic formula describing the $\gamma$-ray spectrum by parameterizing important effects near the pion threshold using an approximate description developed by Dermer (1986a,b), which combines isobaric (Stecker 1970) and scaling models (Badhwar et al. 1977; Stephens \& Badhwar 1981) of the hadronic reaction. Using this formalism, an analytic $\mathcal{F}_{\gamma}-F_{X}$ scaling relation is derived in the framework of a simple scenario of spatial distribution of CRp (Sect. 2.3). Finally, Sect. 2.4 deals with radio and X-ray emission of secondary electrons being produced by decaying charged pions.

\subsection{Definitions}

Throughout the paper we use the following definitions for the differential source function $q(\boldsymbol{r}, E)$, the emissivity $j(\boldsymbol{r}, E)$ and the volume integrated quantities, respectively:

$$
\begin{array}{lr}
q(\boldsymbol{r}, E)=\frac{\mathrm{d} N}{\mathrm{~d} t \mathrm{~d} V \mathrm{~d} E}, & j(\boldsymbol{r}, E)=E q(\boldsymbol{r}, E), \\
Q(E)=\int \mathrm{d} V q(\boldsymbol{r}, E), & J(E)=E Q(E),
\end{array}
$$

where $N$ denotes the integrated number of particles. From the source function the integrated number density production rate of particles $\lambda(\boldsymbol{r})$, the number of particles produced per unit time interval within a certain volume, $\mathcal{L}$, and the particle flux $\mathcal{F}$ can be derived. The definitions of the energy weighted quantities are denoted on the right hand side, respectively,

$$
\begin{array}{ll}
\lambda(\boldsymbol{r})=\int \mathrm{d} E q(\boldsymbol{r}, E), & \Lambda(\boldsymbol{r})=\int \mathrm{d} E E q(\boldsymbol{r}, E), \\
\mathcal{L}=\int \mathrm{d} V \lambda(\boldsymbol{r}), & L=\int \mathrm{d} V \Lambda(\boldsymbol{r}), \\
\mathcal{F}=\frac{\mathcal{L}}{4 \pi D^{2}}, & F=\frac{L}{4 \pi D^{2}} .
\end{array}
$$

\section{2. $\gamma$-ray spectrum from hadronic CRp interactions}

\subsubsection{Cosmic ray proton population}

The differential number density distribution of a CRp population can be described by a power-law in momentum $p_{\mathrm{p}}$,

$f_{\mathrm{p}}\left(\boldsymbol{r}, p_{\mathrm{p}}\right) \mathrm{d} p_{\mathrm{p}} \mathrm{d} V=\tilde{n}_{\mathrm{CRp}}(\boldsymbol{r})\left(\frac{p_{\mathrm{p}} c}{\mathrm{GeV}}\right)^{-\alpha_{\mathrm{p}}}\left(\frac{c \mathrm{~d} p_{\mathrm{p}}}{\mathrm{GeV}}\right) \mathrm{d} V$,

where the tilde indicates that $\tilde{n}_{\mathrm{CRp}}$ is not a real CRp number density while it exhibits those dimensions. We choose the normalization $\tilde{n}_{\mathrm{CRp}}(\boldsymbol{r})$ in such a way that the kinetic CRp energy density $\varepsilon_{\mathrm{CRp}}(\boldsymbol{r})$ is proportional to the thermal energy density $\varepsilon_{\mathrm{th}}(\boldsymbol{r})$ of the ICM,

$$
\begin{aligned}
\varepsilon_{\mathrm{CRp}}(\boldsymbol{r}) & =X_{\mathrm{CRp}}(\boldsymbol{r}) \varepsilon_{\mathrm{th}}(\boldsymbol{r})=\int_{0}^{\infty} \mathrm{d} p f_{\mathrm{p}}\left(\boldsymbol{r}, p_{\mathrm{p}}\right) E_{\mathrm{kin}}\left(p_{\mathrm{p}}\right) \\
& =\frac{\tilde{n}_{\mathrm{CRp}}(\boldsymbol{r}) m_{\mathrm{p}} c^{2}}{2\left(\alpha_{\mathrm{p}}-1\right)}\left(\frac{m_{\mathrm{p}} c^{2}}{\mathrm{GeV}}\right)^{1-\alpha_{\mathrm{p}}} \mathcal{B}\left(\frac{\alpha_{\mathrm{p}}-2}{2}, \frac{3-\alpha_{\mathrm{p}}}{2}\right) .
\end{aligned}
$$

The kinetic energy of CRp $E_{\text {kin }}$ and the thermal energy density of the ICM $\varepsilon_{\text {th }}$ are given by

$$
\begin{aligned}
& E_{\mathrm{kin}}\left(p_{\mathrm{p}}\right)=\sqrt{p_{\mathrm{p}}^{2} c^{2}+m_{\mathrm{p}}^{2} c^{4}}-m_{\mathrm{p}} c^{2}, \\
& \varepsilon_{\mathrm{th}}(\boldsymbol{r})=\frac{3}{2} d_{\mathrm{e}} n_{\mathrm{e}}(\boldsymbol{r}) k T_{\mathrm{e}}(\boldsymbol{r}), \\
& \text { where } d_{\mathrm{e}}=1+\frac{1-\frac{3}{4} X_{\mathrm{He}}}{1-\frac{1}{2} X_{\mathrm{He}}}
\end{aligned}
$$

counts the number of particles per electron in the ICM using the primordial ${ }^{4} \mathrm{He}$ mass fraction $X_{\mathrm{He}}=0.24$, and $\mathcal{B}(a, b)$ denotes the beta-function (Abramowitz \& Stegun 1965). The functional dependence of the CRp scaling parameter $X_{\mathrm{CRp}}(\boldsymbol{r})$ is a priori unknown. In order to draw astrophysical conclusions for the 


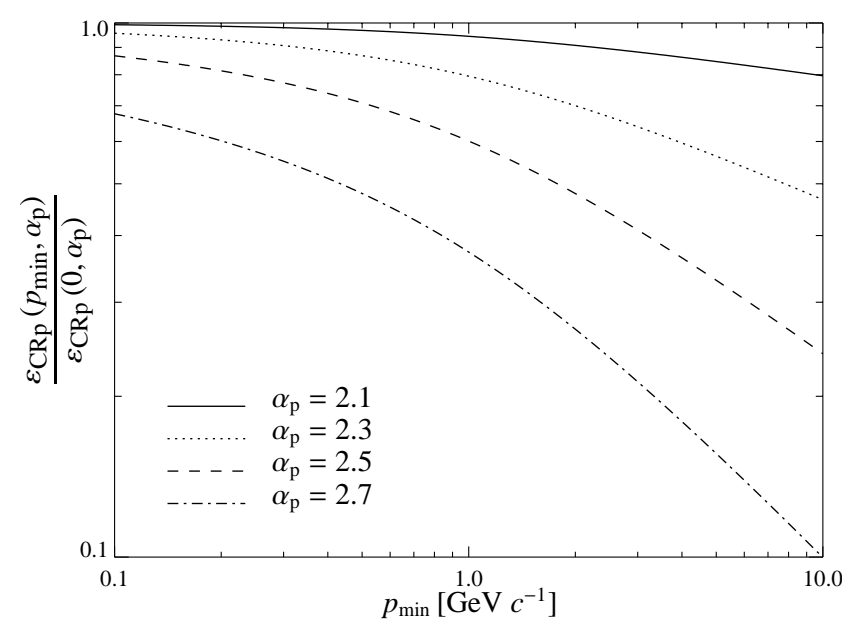

Fig. 1. The ratio of CRp energy densities $\varepsilon_{\mathrm{CRp}}\left(p_{\min }, \alpha_{\mathrm{p}}\right)$ with and without a lower cutoff $p_{\min }$ in the CRp number density distribution function as a function of $p_{\min }$ for different values of the CRp spectral index $\alpha_{\mathrm{p}}$ (see Eq. (15)). For CRp the kinematically allowed threshold in order to produce $\pi^{0}$-mesons hadronically is given by $p_{\mathrm{thr}}=0.78 \mathrm{GeV} c^{-1}$.

CRp population in clusters of galaxies, we adopt three different models for the spatial distribution of CRp later on in Sect. 3.2.

In contrast to relativistic electrons which loose their energy on relatively short time scales compared to the Hubble time through synchrotron emission in cluster magnetic fields and IC scattering with photons of the microwave background, the dominant energy loss mechanisms of CRp are electronic excitations in the plasma (Enßlin et al. 1997), defining a cooling time (Gould 1972)

$$
\begin{aligned}
t_{\mathrm{ee}} & =\left[-\frac{1}{\gamma_{\mathrm{p}}}\left(\frac{\mathrm{d} \gamma_{\mathrm{p}}}{\mathrm{d} t}\right)_{\mathrm{ee}}\right]^{-1} \\
& =\frac{m_{\mathrm{e}} c^{3} m_{\mathrm{p}} \beta_{\mathrm{p}} \gamma_{\mathrm{p}}}{4 \pi e^{4} n_{\mathrm{e}}}\left[\ln \left(\frac{2 \gamma_{\mathrm{p}} m_{\mathrm{e}} c^{2} \beta_{\mathrm{p}}^{2}}{\hbar \omega_{\mathrm{pl}}}\right)-\frac{\beta_{\mathrm{p}}^{2}}{2}\right]^{-1},
\end{aligned}
$$

where $\beta_{\mathrm{p}} c$ denotes the velocity of the proton, $\gamma_{\mathrm{p}}$ its relativistic Lorentz factor, and $\omega_{\mathrm{pl}}=\left(4 \pi e^{2} n_{\mathrm{e}} / m_{\mathrm{e}}\right)^{1 / 2}$ the plasma frequency. Inserting typical values for cooling flows yields a lower cutoff on the CRp momentum

$p_{\text {min }}=\beta_{\mathrm{p}} \gamma_{\mathrm{p}} m_{\mathrm{p}} c \simeq 0.2\left(\frac{t_{\mathrm{age}}}{\mathrm{Gyr}}\right)\left(\frac{n_{\mathrm{e}}}{10^{-2} \mathrm{~cm}^{-3}}\right) \mathrm{GeV} c^{-1}$.

In general, this gives rise to a spatially dependent cutoff of the CRp momentum which increases with time.

In order not to rely on too many assumptions, we do not impose a specific momentum cutoff which is possible since the spectral index $\alpha_{\mathrm{p}}$ varies in our model in between 2 and 3 . Instead, we quantify the influence of a lower cutoff $p_{\min }$ on the population of $\mathrm{CRp}$ by taking the ratio of CRp energy densities $\varepsilon_{\mathrm{CRp}}\left(p_{\min }, \alpha_{\mathrm{p}}\right)$ with and without a lower cutoff. This ratio as shown in Fig. 1 can be written using the definition for the normalized lower CRp momentum cutoff $\tilde{p}=\frac{p_{\min }}{m_{\mathrm{p}} c}$,

$$
\frac{\varepsilon_{\mathrm{CRp}}\left(\tilde{p}, \alpha_{\mathrm{p}}\right)}{\varepsilon_{\mathrm{CRp}}\left(0, \alpha_{\mathrm{p}}\right)}=\frac{\mathcal{B}_{x}\left(\frac{\alpha_{\mathrm{p}}-2}{2}, \frac{3-\alpha_{\mathrm{p}}}{2}\right)+2 \tilde{p}^{1-\alpha_{\mathrm{p}}}\left(\sqrt{1+\tilde{p}^{2}}-1\right)}{\mathcal{B}\left(\frac{\alpha_{\mathrm{p}}-2}{2}, \frac{3-\alpha_{\mathrm{p}}}{2}\right)}
$$

where $\mathcal{B}_{x}(a, b)$ denotes the incomplete beta-function (Abramowitz \& Stegun 1965) with $x=\left(1+\tilde{p}^{2}\right)^{-1}$. Combining Fig. 1 and Eq. (14) demonstrates the small influence of Coulomb cooling to the CRp energy density within cooling flows.

\subsubsection{Fireball model}

The CRp interact hadronically with the thermal background gas and produce pions with relative multiplicities $\xi_{\pi^{0}}=\frac{1}{2} \xi_{\pi^{ \pm}}$ according to isospin symmetry and assuming thermal equilibrium of the pion cloud in the center of mass (Fermi 1950). The charged pions decay into secondary electrons (and neutrinos) and the neutral pions into $\gamma$-rays:

$$
\begin{aligned}
& \pi^{ \pm} \rightarrow \mu^{ \pm}+v_{\mu} / \bar{v}_{\mu} \rightarrow \mathrm{e}^{ \pm}+v_{\mathrm{e}} / \bar{v}_{\mathrm{e}}+v_{\mu}+\bar{v}_{\mu} \\
& \pi^{0} \rightarrow 2 \gamma
\end{aligned}
$$

Only CRp above the kinematic threshold $p_{\text {thr }}=0.78 \mathrm{GeV} c^{-1}$ are able to produce pions hadronically and are therefore visible through their decay products in both the $\gamma$-ray and radio bands via radiative processes. Only the CRp population above this threshold is constrained by this work while the lower energy part of this population in general can not be limited by only considering hadronic interactions.

In the high-energy limit for CRp $\left(E_{\mathrm{p}} \gg m_{\mathrm{p}} c^{2}\right)$ the pion source function resulting from hadronic CRp-p interactions can be calculated following Mannheim \& Schlickeiser (1994) to be

$$
\begin{aligned}
q_{\pi^{0}}\left(\boldsymbol{r}, E_{\pi^{0}}\right) \mathrm{d} E_{\pi^{0}} \mathrm{~d} V \approx & 2^{3} \bar{\sigma}_{\mathrm{pp}} c n_{\mathrm{N}}(\boldsymbol{r}) \frac{\tilde{n}_{\mathrm{CRp}}(\boldsymbol{r})}{\mathrm{GeV}} \\
& \times\left(\frac{6 E_{\pi^{0}}}{\mathrm{GeV}}\right)^{-\alpha_{\gamma}} \mathrm{d} E_{\pi^{0}} \mathrm{~d} V,
\end{aligned}
$$

where $\alpha_{\gamma}=4 / 3\left(\alpha_{\mathrm{p}}-1 / 2\right), \bar{\sigma}_{\mathrm{pp}}=32$ mbarn is the inelastic p-p cross section, and $n_{\mathrm{N}}(\boldsymbol{r})=d_{\mathrm{tar}} n_{\mathrm{e}}(\boldsymbol{r})=n_{\mathrm{e}}(\boldsymbol{r}) /\left(1-\frac{1}{2} X_{\mathrm{He}}\right)$ is the target nucleon density in the ICM. The $\pi^{0}$-decay induced omnidirectional (i.e. integrated over $4 \pi$ solid angle) differential $\gamma$-ray source function can be calculated in this energy regime assuming the decay products are distributed isotropically in their rest frame, yielding

$$
\begin{aligned}
q_{\gamma}\left(\boldsymbol{r}, E_{\gamma}\right) & =2 \int_{E_{\gamma}+\frac{m_{\pi}^{2} c^{4}}{4 E_{\gamma}}}^{\infty} \mathrm{d} E_{\pi^{0}} \frac{q_{\pi^{0}}\left(\boldsymbol{r}, E_{\pi^{0}}\right)}{\sqrt{E_{\pi^{0}}^{2}-m_{\pi^{0}}^{2} c^{4}}} \\
& =2^{3} \bar{\sigma}_{\mathrm{pp}} c n_{\mathrm{N}}(\boldsymbol{r}) \frac{\tilde{n}_{\mathrm{CRp}}(\boldsymbol{r})}{\mathrm{GeV}}\left(\frac{6 m_{\pi^{0}} c^{2}}{\mathrm{GeV}}\right)^{-\alpha_{\gamma}} \mathcal{B}_{x}\left(\frac{\alpha_{\gamma}}{2}, \frac{1}{2}\right) \\
\text { where } x & =\left(\frac{4 E_{\gamma} m_{\pi^{0}} c^{2}}{4 E_{\gamma}^{2}+m_{\pi^{0}}^{2} c^{4}}\right)^{2}
\end{aligned}
$$

Owing to Lorentz symmetry, this formula is valid for both limiting energy regimes, $E_{\gamma} \gg m_{\pi^{0}} c^{2} / 2$ and $E_{\gamma} \ll m_{\pi^{0}} c^{2} / 2$. Because of an incomplete accounting of physical processes at the threshold of pion production like the velocity distribution of CRp and momentum dependent inelastic CRp-p cross section, Eq. (17) overestimates the number of $\gamma$-rays for energies around $E_{\gamma} \simeq m_{\pi^{0}} c^{2} / 2$. 


\subsubsection{Dermer's model}

In order to make detailed predictions for the $\pi^{0}$-decay induced $\gamma$-ray spectrum, more realistic effects near the $\pi^{0}$-production threshold have to be included. This was done by using the code COSMOCR originally designed for cosmic ray studies by Miniati (2001). The underlying $\Delta_{3 / 2}$-isobaric model was shown to work well at low proton energies (Stecker 1970). It assumes the CRp-p interaction to be mediated by the excitation of the $\Delta_{3 / 2}$-resonance which subsequently decays into two protons and a $\pi^{0}$-meson. The production spectrum of secondary $\pi^{0}$-mesons is given by a convolution of the normalized $\Delta_{3 / 2^{-}}$ isobar mass spectrum represented by a Breit-Wigner distribution with the energy distribution function. The scaling model used at high energies (Stephens \& Badhwar 1981) uses Lorentz invariant cross sections for charged and neutral pion production in p-p interactions inferred from accelerator data. COSMOCR includes also the contribution of the two main kaon decay modes to secondary pion spectra (following Moskalenko \& Strong 1998) which are $K^{ \pm} \rightarrow \mu^{ \pm}+v_{\mu} / \bar{v}_{\mu}(63.5 \%)$ and $K^{ \pm} \rightarrow$ $\pi^{0}+\pi^{ \pm}(21.2 \%)$ where the latter channel also contributes to the $\gamma$-ray source function.

In order to derive an analytic formula describing the omnidirectional differential $\gamma$-ray source function over the energy range shown in Fig. 2, we keep the behavior of the spectrum in the fireball model for $E_{\gamma} \gg m_{\pi^{0}} c^{2} / 2$ and parameterize the detailed physics at the $\pi^{0}$-threshold by the shape parameter $\delta_{\gamma}$ which smoothly joins the two power laws to the asymptotic expansion of the $\mathcal{B}$-function of Eq. (17), yielding

$$
\begin{aligned}
& q_{\gamma}\left(\boldsymbol{r}, E_{\gamma}\right) \mathrm{d} E_{\gamma} \mathrm{d} V \simeq \sigma_{\mathrm{pp}} c n_{\mathrm{N}}(\boldsymbol{r}) \xi^{2-\alpha_{\gamma}} \frac{\tilde{n}_{\mathrm{CRp}}(\boldsymbol{r})}{\mathrm{GeV}} \\
& \quad \times \frac{4}{3 \alpha_{\gamma}}\left(\frac{m_{\pi^{0}} c^{2}}{\mathrm{GeV}}\right)^{-\alpha_{\gamma}}\left[\left(\frac{2 E_{\gamma}}{m_{\pi^{0}} c^{2}}\right)^{\delta_{\gamma}}+\left(\frac{2 E_{\gamma}}{m_{\pi^{0}} c^{2}}\right)^{-\delta_{\gamma}}\right]^{-\alpha_{\gamma} / \delta_{\gamma}} \mathrm{d} E_{\gamma} \mathrm{d} V .
\end{aligned}
$$

The scaling behavior in the high-energy limit of Dermer's model can be described by a constant pion multiplicity $\xi=2$ characterizing the two leading pion jets leaving the interaction site in direction of the incident protons diametrically and carrying the high longitudinal momenta owing to Lorentz contraction of the interacting nuclei in the center of mass system and Heisenberg's uncertainty relation (Nachtmann 1990). This assumption of constant pion multiplicity of the scaling model is in contrast to the fireball model (Mannheim \& Schlickeiser 1994), which assumes a state of hot quark-gluon plasma in thermal equilibrium after the hadronic interaction subsequently ablating pions with multiplicities $\xi_{\pi^{0}} \simeq\left[\left(E_{\mathrm{p}}-E_{\mathrm{th}}\right) / \mathrm{GeV}\right]^{1 / 4}$, where $E_{\mathrm{th}}=1.22 \mathrm{GeV}$ denotes the threshold energy for pion production.

The $\gamma$-ray source function peaks at the energy of $m_{\pi^{0}} c^{2} / 2 \simeq$ $67.5 \mathrm{MeV}$. It is well known, that the asymptotic slope of the $\gamma$-ray spectrum, characterized by its spectral index $\alpha_{\gamma}$, reproduces the spectral index of the population of CRp, $\alpha_{\gamma}=\alpha_{\mathrm{p}}$ (Dermer 1986b). This is again in contrast to the fireball model which predicts a steeper asymptotic slope in the $\gamma$-ray spectrum for $\alpha_{\mathrm{p}}>2$, amounting to $\alpha_{\gamma}=4 / 3\left(\alpha_{\mathrm{p}}-1 / 2\right)$. In the following we restrict ourselves to Dermer's model because it is better motivated by accelerator data.
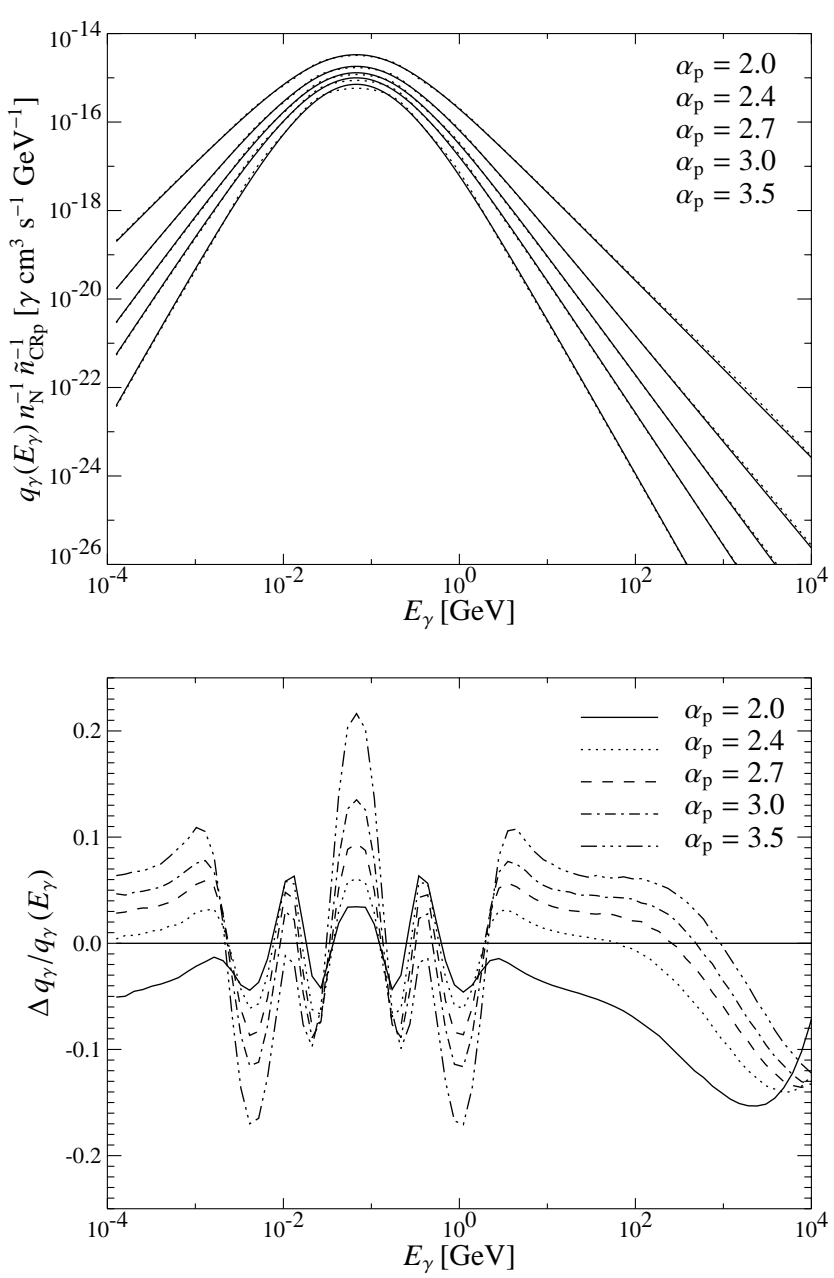

Fig. 2. a) The omnidirectional (i.e. integrated over $4 \pi$ solid angle) differential $\gamma$-ray source function $q_{\gamma}\left(E_{\gamma}\right)$ normalized by the target number density $n_{\mathrm{N}}(\boldsymbol{r})$ and CRp normalization $\tilde{n}_{\mathrm{CRp}}(\boldsymbol{r})$ in order to be independent of the spatial dependence of any specific model. The dotted lines show the simulated $\gamma$-ray spectra while the solid curves represent our models given by Eq. (19) with the spectral indices from top to bottom, $\alpha_{\mathrm{p}} \in\{2.0,2.4,2.7,3.0,3.5\}$. b) Relative deviation of our analytic approach to simulated $\gamma$-ray spectra.

By comparing the logarithm of the $\gamma$-ray source function of Eq. (19) to numerically calculated spectra using COSMOCR we recognized that the influence of the detailed physics at the threshold together with the kaon contribution can be modeled in our semi-analytic approach in Eq. (19) by self-consistent scaling relations for the shape parameter $\delta_{\gamma}$ and the effective inelastic p-p cross section $\sigma_{\mathrm{pp}}$ including the kaon decay modes. The shape parameter $\delta_{\gamma}$ scales with the spectral index of the $\gamma$-ray spectrum as

$\delta_{\gamma}=0.14 \alpha_{\gamma}^{-1.6}+0.44$,

which models the functional behavior of the spectrum (compare Fig. 2). The effective cross section $\sigma_{\mathrm{pp}}$ also depends on $\alpha_{\gamma}$ which can be modeled by

$\sigma_{\text {pp }}=32 \times\left(0.96+\mathrm{e}^{4.4-2.4 \alpha_{\gamma}}\right)$ mbarn.

On the one hand, the enhanced contribution to the normalization of the $\gamma$-ray source function $q_{\gamma}\left(E_{\gamma}\right)$ for flat spectral 
indices $\alpha_{\gamma}$ is due to the larger contribution of the channel $p+p \rightarrow K^{ \pm}+X$ relative to $p+p \rightarrow \pi^{ \pm}+X$ for larger energies, approaching asymptotically a value of $27 \%$ at energies larger than $1 \mathrm{TeV}$ (Miniati 2001) which we did not account for a priori in our simple model. Secondly, this scaling behavior also includes higher order contributions to the effective pion multiplicity for harder spectra characterized by a lower spectral index $\alpha_{\gamma}$.

The effective description of the spectrum with the smooth peak characterized by the shape parameter $\delta_{\gamma}$ starts to fail for very steep spectra of $\alpha_{\gamma}>3.5$ where relativistic kinematics at the threshold plays a crucial role. Then the higher number of decaying low energetic $\pi^{0}$-mesons results in a more concentrated peak on top of the boosted broader distribution of decaying highly-energetic pions. The lower panel of Fig. 2 shows that the relative deviation of the semi-analytic approach of Eq. (19) to the simulated $\gamma$-ray spectra amounts below 0.2 for the spectral range shown in Fig. 2, which is sufficient for the purpose of our work.

\subsection{Energy band integrated $\gamma$-ray luminosity: Analytic $\mathcal{F}_{\gamma}-F_{X}$ scaling relation}

In the following, we derive an analytic $\mathcal{F}_{\gamma}-F_{X}$ scaling relation which should serve as an approximate estimate for a given cluster of galaxies. As a simple scenario we choose the CRp energy density to be a constant fraction of the thermal energy density, $\varepsilon_{\mathrm{CRp}}(\boldsymbol{r})=X_{\mathrm{CRp}} \varepsilon_{\mathrm{th}}(\boldsymbol{r})$. However, this is not a fundamental constraint for this scaling relation. Any other spatial dependency for the CRp scaling parameter $X_{\mathrm{CRp}}$ may be substituted instead of the assumed one.

The bolometric X-ray emission of the hot thermal intra cluster electrons is given by the cooling function for thermal bremsstrahlung (Rybicki \& Lightman 1979),

$$
\begin{aligned}
& \begin{array}{l}
\Lambda_{\mathrm{X}}\left[n_{\mathrm{e}}(\boldsymbol{r}), T_{\mathrm{e}}(\boldsymbol{r})\right]=\Lambda_{0} n_{\mathrm{e}}(\boldsymbol{r})^{2} \sqrt{k T_{\mathrm{e}}(\boldsymbol{r})}, \\
\text { with } \Lambda_{0}=\left(\frac{2 \pi}{3 m_{\mathrm{e}}}\right)^{1 / 2} \frac{2^{5} \pi e^{6} d_{\mathrm{tar}}}{3 h m_{\mathrm{e}} c^{3}} Z^{2} \bar{g}_{\mathrm{B}}\left(T_{\mathrm{e}}\right) \\
\simeq 6.62 \times 10^{-24} \mathrm{erg} \mathrm{s}^{-1} \mathrm{~cm}^{3} \mathrm{keV}^{-1 / 2},
\end{array}
\end{aligned}
$$

where $n_{\mathrm{e}}$ is the electron number density, $T_{\mathrm{e}}$ the temperature, $d_{\mathrm{tar}}$ is the nucleon density in the ICM relative to the electrons for primordial element composition, $Z$ the charge number ${ }^{1}$ and $\bar{g}_{\mathrm{B}} \simeq 1.2$ is the frequency and velocity averaged Gaunt factor.

In order to obtain the integrated $\gamma$-ray source density $\lambda_{\gamma}$ for pion decay induced $\gamma$-rays the $\gamma$-ray source function $q_{\gamma}\left(\boldsymbol{r}, E_{\gamma}\right)$ in Eq. (19) can be integrated over an energy interval yielding

$$
\begin{aligned}
\lambda_{\gamma}\left(\boldsymbol{r}, E_{1}, E_{2}\right) & =\int_{E_{1}}^{E_{2}} \mathrm{~d} E_{\gamma} q_{\gamma}\left(\boldsymbol{r}, E_{\gamma}\right) \\
& =A_{\gamma}\left(\alpha_{\mathrm{p}}\right) N_{\gamma}\left(\alpha_{\mathrm{p}}\right) X_{\mathrm{CRp}} n_{\mathrm{e}}^{2}(\boldsymbol{r}) k T_{\mathrm{e}}(\boldsymbol{r}),
\end{aligned}
$$

${ }^{1}$ Setting $Z^{2}=1$ in Eq. (23) is correct for a plasma of primordial element composition which consists of hydrogen and helium only, because $\left\langle n_{\mathrm{N}} Z^{2}\right\rangle=n_{\mathrm{N}}$ in this case. This is a reasonable approximation owing to the small contamination of heavier elements in the ICM. where $A_{\gamma}\left(\alpha_{\mathrm{p}}\right)=\frac{\sigma_{\mathrm{pp}}\left(\alpha_{\mathrm{p}}\right) d_{\mathrm{e}} d_{\mathrm{tar}} m_{\pi^{0}} c}{\mathrm{GeV} m_{\mathrm{p}}}$,

$N_{\gamma}\left(\alpha_{\mathrm{p}}\right)=\frac{\left(\frac{m_{\pi^{0}} c^{2}}{\mathrm{GeV}}\right)^{-\alpha_{\gamma}}\left[\mathcal{B}_{x}\left(\frac{\alpha_{\gamma}+1}{2 \delta_{\gamma}}, \frac{\alpha_{\gamma}-1}{2 \delta_{\gamma}}\right)\right]_{x_{1}}^{x_{2}}\left(\alpha_{\mathrm{p}}-1\right)}{\left(\frac{m_{\mathrm{p}} c^{2}}{\mathrm{GeV}}\right)^{1-\alpha_{\mathrm{p}}} \mathcal{B}\left(\frac{\alpha_{\mathrm{p}}-2}{2}, \frac{3-\alpha_{\mathrm{p}}}{2}\right) 2^{\alpha_{\gamma}-2} \alpha_{\gamma} \delta_{\gamma}}$,

and $x_{i}=\left[1+\left(\frac{m_{\pi^{0}} c^{2}}{2 E_{i}}\right)^{2 \delta_{\gamma}}\right]^{-1}$ for $i \in\{1,2\}$.

Here we introduced the abbreviation

$[f(x)]_{x_{1}}^{x_{2}}=f\left(x_{2}\right)-f\left(x_{1}\right)$.

Assuming Dermer's model the $\gamma$-ray spectral index scales as $\alpha_{\gamma}=\alpha_{\mathrm{p}}$ in contrast to the fireball model where $\alpha_{\gamma}=$ $4 / 3\left(\alpha_{\mathrm{p}}-1 / 2\right)$. The shape parameter $\delta_{\gamma}$ is given by the $\alpha_{\gamma}-\delta_{\gamma}$ scaling relation in Eq. (20) which strictly holds for Dermer's model, but should also be valid for the extended fireball model.

Comparing the integrated $\gamma$-ray source density $\lambda_{\gamma}\left(E_{1}, E_{2}\right)$ of Eq. (25) to that of thermal bremsstrahlung (Eq. (22)) we obtain an analytic $\mathcal{F}_{\gamma}-F_{X}$ scaling relation for the ratio of $\gamma$-ray fluxes $\mathcal{F}_{\gamma}$ and bolometric X-ray fluxes $F_{\mathrm{X}}$,

$\frac{\mathcal{F}_{\gamma}\left(E_{1}<E<E_{2}\right)}{F_{\mathrm{X}}^{\mathrm{bol}} \mathrm{erg}^{-1}}=\frac{A_{\gamma}\left(\alpha_{\mathrm{p}}\right) N_{\gamma}\left(\alpha_{\mathrm{p}}\right)}{\Lambda_{0} \mathrm{keV}^{-1 / 2} \mathrm{erg}^{-1}}\left(\frac{\left\langle k T_{\mathrm{e}}\right\rangle}{\mathrm{keV}}\right)^{1 / 2} X_{\mathrm{CRp}}$,

where the prefactor is appropriately scaled yielding a dimensionless number which consists of $A_{\gamma}\left(\alpha_{\mathrm{p}}\right)$ (Eq. (27)), $N_{\gamma}\left(\alpha_{\mathrm{p}}\right)$ (Eq. (28)), and $\Lambda_{0}$ (Eq. (23)). The $\mathcal{F}_{\gamma}-F_{\mathrm{X}}$ ratio scales linearly with the scaling parameter $X_{\mathrm{CRp}}$ given by Eq. (7) and is independent of the underlying cosmology, however not of redshift due to the K-correction. Inferred values for the expected $\gamma$-ray flux $\mathcal{F}_{\gamma}$ are consistent with those obtained by Enßlin et al. (1997) for the spectral index of our Galaxy $\alpha_{\gamma}=2.7$.

\subsection{Stationary spectrum of hadronically originating secondary electrons}

This section is based on a formalism developed in Dolag \& Enßlin (2000). The steady-state CRe spectrum is governed by injection of secondaries and cooling processes so that it can be described by the continuity equation

$\frac{\partial}{\partial E_{\mathrm{e}}}\left(\dot{E}_{\mathrm{e}}\left(\boldsymbol{r}, E_{\mathrm{e}}\right) f_{\mathrm{e}}\left(\boldsymbol{r}, E_{\mathrm{e}}\right)\right)=q_{\mathrm{e}}\left(\boldsymbol{r}, E_{\mathrm{e}}\right)$.

For $\dot{E}_{\mathrm{e}}(\boldsymbol{r}, p)<0$ this equation is solved by

$f_{\mathrm{e}}\left(\boldsymbol{r}, E_{\mathrm{e}}\right)=\frac{1}{\mid \dot{E}_{\mathrm{e}}\left(\boldsymbol{r}, E_{\mathrm{e}}\right)} \int_{E_{\mathrm{e}}}^{\infty} \mathrm{d} E_{\mathrm{e}}^{\prime} q_{\mathrm{e}}\left(\boldsymbol{r}, E_{\mathrm{e}}^{\prime}\right)$.

The cooling of the radio emitting CRe is dominated by synchrotron and inverse Compton losses giving

$-\dot{E}_{\mathrm{e}}\left(\boldsymbol{r}, E_{\mathrm{e}}\right)=\frac{4 \sigma_{\mathrm{T}} c}{3 m_{\mathrm{e}}^{2} c^{4}}\left(\frac{B^{2}(\boldsymbol{r})}{8 \pi}+\frac{B_{\mathrm{CMB}}^{2}}{8 \pi}\right) E_{\mathrm{e}}^{2}$,

where $\sigma_{\mathrm{T}}$ is the Thomson cross section, $B(\boldsymbol{r})$ is the local magnetic field strength and $B_{\mathrm{CMB}}^{2} /(8 \pi)$ is the energy density of the 
cosmic microwave background expressed by an equivalent field strength $B_{\mathrm{CMB}}=3.24(1+z)^{2} \mu \mathrm{G}$. The CRe population above a $\mathrm{GeV}$ is therefore described by a power-law spectrum

$f_{\mathrm{e}}\left(\boldsymbol{r}, E_{\mathrm{e}}\right)=\frac{\tilde{n}_{\mathrm{CRe}}(\boldsymbol{r})}{\mathrm{GeV}}\left(\frac{E_{\mathrm{e}}}{\mathrm{GeV}}\right)^{-\alpha_{\mathrm{e}}}$,

$\tilde{n}_{\mathrm{CRe}}(\boldsymbol{r})=\frac{2^{7} \pi 16^{-\left(\alpha_{\mathrm{e}}-1\right)} A_{\mathrm{mod}}}{\alpha_{\mathrm{e}}-2} \frac{\sigma_{\mathrm{pp}} m_{\mathrm{e}}^{2} c^{4}}{\sigma_{\mathrm{T}} \mathrm{GeV}} \frac{n_{\mathrm{N}}(\boldsymbol{r}) \tilde{n}_{\mathrm{CRp}}(\boldsymbol{r})}{B^{2}(\boldsymbol{r})+B_{\mathrm{CMB}}^{2}}$,

$\alpha_{\mathrm{e}}= \begin{cases}\alpha_{\mathrm{p}}+1 & \text { in Dermer's model } \\ \frac{4}{3} \alpha_{\mathrm{p}}+\frac{1}{3} & \text { in the fireball model }\end{cases}$

$A_{\text {mod }}= \begin{cases}1 & \text { in Dermer's model, } \\ 3\left(\frac{3}{2}\right)^{-\left(\alpha_{\mathrm{e}}-1\right)} & \text { in the fireball model. }\end{cases}$

For the sake of consistency, we use Dermer's model throughout the paper where the effective cross section $\sigma_{\mathrm{pp}}$ is given by Eq. (21) in contrast to the fireball model where $\sigma_{\mathrm{pp}}=$ 32 mbarn. The approach of the scaling relation of Eq. (21) is approximately valid for CRe although the decay channels of charged kaons provide a stronger contribution to the $\pi^{ \pm}$branching ratio relative to $\pi^{0}$-mesons resulting also in slightly higher injection rates for electrons and positrons. Differences in normalization and radio brightness morphology due to the different models governing the CRp-p interaction are small and irrelevant for our conclusions.

\subsubsection{Synchrotron emission of secondary electrons}

The synchrotron emissivity $j_{v}$ at frequency $v$ and per steradian of a power law distribution of CRe (Eq. (35)) in an isotropic distribution of magnetic fields and electrons within the halo volume (Eq. (6.36) in Rybicki \& Lightman 1979), is obtained after averaging over an isotropic distribution of electron pitch angles yielding

$j_{v}(\boldsymbol{r})=c_{2}\left(\alpha_{\mathrm{e}}\right) \tilde{n}_{\mathrm{CRe}}(\boldsymbol{r}) B(\boldsymbol{r})^{\alpha_{v}+1}\left(\frac{v}{c_{1}}\right)^{-\alpha_{v}}$

with $c_{1}=3 e \mathrm{GeV}^{2} /\left(2 \pi m_{\mathrm{e}}^{3} c^{5}\right)$,

$c_{2}\left(\alpha_{\mathrm{e}}\right)=\frac{\sqrt{3 \pi}}{32 \pi} \frac{e^{3}}{m_{\mathrm{e}} c^{2}} \frac{\alpha_{\mathrm{e}}+\frac{7}{3}}{\alpha_{\mathrm{e}}+1} \frac{\Gamma\left(\frac{3 \alpha_{\mathrm{e}}-1}{12}\right) \Gamma\left(\frac{3 \alpha_{\mathrm{e}}+7}{12}\right) \Gamma\left(\frac{\alpha_{\mathrm{e}}+5}{4}\right)}{\Gamma\left(\frac{\alpha_{\mathrm{e}}+7}{4}\right)}$,

where $\Gamma(a)$ denotes the $\Gamma$-function (Abramowitz \& Stegun 1965) and $\alpha_{v}=\left(\alpha_{\mathrm{e}}-1\right) / 2=\alpha_{\mathrm{p}} / 2$ in Dermer's model. In our models the magnetic field $B(r)$ was assumed to be spherically symmetric on cluster core scales and to follow the electron density $n_{\mathrm{e}}(r)$ (Dolag et al. 1999, 2001):

$B(r)=B_{0}\left[\frac{n_{\mathrm{e}}(r)}{n_{\mathrm{e}}(0)}\right]^{\alpha_{B}}$,

where $B_{0}$ and $\alpha_{B}$ are free parameters in our model. Assuming the radio emissivity $j_{v}(\boldsymbol{r})$ in Eq. (39) to be only a function of radius, then the line of sight integration yields the surface brightness of the radio halo

$S_{v}\left(r_{\perp}\right)=2 \int_{r_{\perp}}^{R} \frac{j_{v}(r) r \mathrm{~d} r}{\sqrt{r^{2}-r_{\perp}^{2}}}$

\subsubsection{Inverse Compton emission of secondary electrons}

The source function $q_{\mathrm{IC}}$ owing to IC scattering of cosmic microwave background (CMB) photons off an isotropic power law distribution of hadronically originating CRe (Eq. (35)) is (derived from Eq. (7.31) in Rybicki \& Lightman 1979, in the case of Thomson scattering),

$$
\begin{aligned}
q_{\mathrm{IC}}\left(\boldsymbol{r}, E_{\gamma}\right) & =\tilde{q}(\boldsymbol{r}) f_{\mathrm{IC}}\left(\alpha_{\mathrm{e}}\right)\left(\frac{m_{\mathrm{e}} c^{2}}{\mathrm{GeV}}\right)^{1-\alpha_{\mathrm{e}}}\left(\frac{E_{\gamma}}{k T_{\mathrm{CMB}}}\right)^{-\left(\alpha_{\nu}+1\right)}, \\
f_{\mathrm{IC}}\left(\alpha_{\mathrm{e}}\right)= & \frac{2^{\alpha_{\mathrm{e}}+3}\left(\alpha_{\mathrm{e}}^{2}+4 \alpha_{\mathrm{e}}+11\right)}{\left(\alpha_{\mathrm{e}}+3\right)^{2}\left(\alpha_{\mathrm{e}}+5\right)\left(\alpha_{\mathrm{e}}+1\right)} \\
& \times \Gamma\left(\frac{\alpha_{\mathrm{e}}+5}{2}\right) \zeta\left(\frac{\alpha_{\mathrm{e}}+5}{2}\right),
\end{aligned}
$$

and $\tilde{q}(\boldsymbol{r})=\frac{8 \pi^{2} r_{\mathrm{e}}^{2} \tilde{n}_{\mathrm{CRe}}(\boldsymbol{r})\left(k T_{\mathrm{CMB}}\right)^{2}}{h^{3} c^{2}}$,

where $\alpha_{v}=\left(\alpha_{\mathrm{e}}-1\right) / 2$ denotes the spectral index, $r_{\mathrm{e}}=$ $e^{2} /\left(m_{\mathrm{e}} c^{2}\right)$ the classical electron radius, $\zeta(a)$ the Riemann $\zeta$-function (Abramowitz \& Stegun 1965), and $\tilde{n}_{\mathrm{CRe}}(\boldsymbol{r})$ is given by Eq. (36). After integrating over the IC emitting volume in the cluster we obtain the particle flux $\mathcal{F}\left(E_{\gamma}\right)$ (see Eqs. (4) and (5)). The same CRe population seen in the radio band via synchrotron emission scatter CMB photons into the hard X-ray regime. In the $\gamma$-ray spectrum, there is a point of equal contribution of the IC spectrum of the CRe showing a decreasing slope of $-\alpha_{v}-1=-\alpha_{\mathrm{p}} / 2-1$ (assuming Dermer's model) and the pion decay induced $\gamma$-ray spectrum being characterized by the rising slope $\alpha_{\gamma}=\alpha_{\mathrm{p}}$ (see Eq. (19)). In the high energy limit $\left(E_{\gamma} \gg m_{\pi^{0}} c^{2} / 2\right)$, the pion decay induced $\gamma$-ray spectrum declines with a slope of $-\alpha_{\gamma}=-\alpha_{\mathrm{p}}$ which is the same as the IC emission for $\alpha_{\mathrm{p}}=2$ and slightly steeper for larger values of $\alpha_{\mathrm{p}}$ (for illustration, see Fig. 3).

\subsection{Summary and outline}

In the previous sections we developed an analytic description to compute the neutral pion decay induced $\gamma$-ray spectrum from a CRp population over a broad range of $\gamma$-ray energies extending from below $\mathrm{MeV}$ up to $\mathrm{TeV}$. Moreover, we presented a formalism of calculating the synchrotron and IC emission from a stationary population of CRe resulting from hadronic CRp interactions of the thermal gas of the ICM. Assuming a constant scaling between kinetic CRp energy density and thermal energy density of the ICM we derived an analytic $\mathcal{F}_{\gamma}-F_{\mathrm{X}}$ scaling relation for the ratio of $\gamma$-ray flux to bolometric X-ray flux to obtain observationally promising cluster candidates for constraining the CRp population. In order to obtain reliable flux estimates we are going to introduce in the following three possible spatial distributions of the CRp, whose population is either in fractional pressure equilibrium with the thermal particle population (as assumed for selecting the clusters), experienced adiabatic compression during the formation of the cooling flow cluster or is shaped by diffusion away from a central source of CRp. By modeling the $\gamma$-ray emission of these particular 


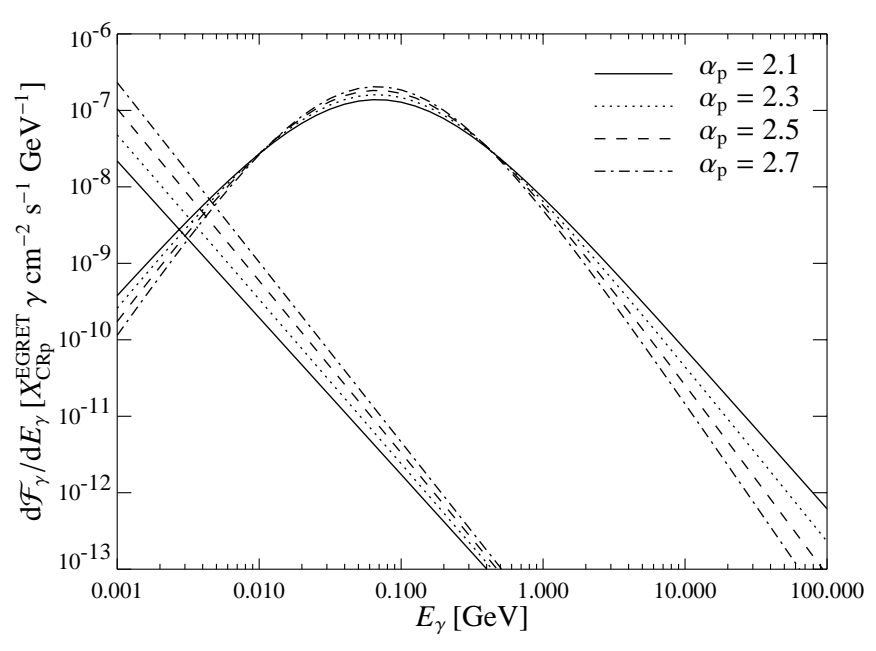

Fig. 3. The simulated differential flux of $\gamma$-rays from Perseus reaching the Earth. Shown are upper limits of the IC emission of secondary CRe (power-laws, assuming zero magnetic field) as well as pion decay induced $\gamma$-ray emission (represented by broad distribution centered on $E_{\text {peak }} \simeq 67.5 \mathrm{MeV}$ ). The normalization of the spectra differing in their values of the CRp spectral index $\alpha_{\gamma}=\alpha_{\mathrm{p}}$ (Dermer's model) depends on the assumed scaling between CRp and thermal energy density. We fix this scaling parameter $X_{\mathrm{CRp}}$ assuming the isobaric model (Sect. 3.2.1) by comparing the integrated flux above $100 \mathrm{MeV}$ to EGRET upper limits (see Reimer et al. 2003).

clusters and comparing to EGRET upper limits we are going to present bounds on the CRp population. Furthermore, we will derive upper bounds on the CRp population by radio synchrotron emission of hadronically originating CRe and will compare azimuthally averaged radio brightness profiles of the the Perseus radio-mini halo and the radio halo of Coma.

\section{Astrophysical application to nearby clusters of galaxies using multi-frequency observations}

\subsection{The expected spectral index $\alpha_{p}$}

The spectral index of the ICM CRp population $\alpha_{\mathrm{p}}$ is not well constrained by observations. However, because galaxy clusters are able to store CRp for cosmological times (Völk et al. 1996; Enßlin et al. 1997; Berezinsky et al. 1997) the spectral index of the global CRp population (allowing for spatial differentiation) is expected to be that of the injection process, if no reacceleration processes modified the spectrum after injection. We discuss briefly different possible CRp sources in galaxy clusters:

Structure formation shock waves have generated most of the thermal energy content of galaxy clusters. Therefore, it is plausible to assume that they also produced most of the CR energy of clusters. Shock acceleration is able to produce momentum power-law particle distributions characterized by a spectral index, which is in the test-particle picture of non-relativistic shock acceleration

$\alpha_{\text {inj }}=\frac{R+2}{R-1}$,

where $R \leq 4$ is the shock compression factor. The lowest spectral indices are therefore generated by the strongest shocks, which are preferentially found in peripheral regions of the clusters (Quilis et al. 1998; Miniati et al. 2000). Thus, harder CRp populations $\left(\alpha_{\mathrm{inj}}=2.0 \ldots 2.5\right)$ are mostly injected into the outskirts of clusters. However, motion of the ICM gas transports them efficiently into the cluster centers (Miniati et al. 2001b).

Injection by radio galaxies: Active galactic nuclei (AGN) are able to produce large amounts of relativistic plasma. The composition of this plasma is not known, however, the presence of CRp is assumed in and supported by many papers. The energetics of AGN is sufficient to inject a significant CRp population into the thermal ICM (Enßlin et al. 1997, 1998b; Colafrancesco \& Blasi 1998; Blasi 1999; Wu et al. 2000) provided CRp are present in the radio plasma and are able to leave it on cosmological short timescales. If the radio plasma releases all its $\mathrm{CRp}$, a moderately flat injection spectrum can be expected (say $\alpha_{\text {inj }} \approx 2.5$ ) since radio emission from radio galaxies indicates flat CRe spectra. If, however, only a small fraction of the CRp is able to leave the radio plasma diffusively, an even flatter spectrum (say $\alpha_{\text {inj }} \approx 2.2$ ) can be expected due to increasing escape probability with momentum (Enßlin 2003).

Supernova Remnants (SNR) are known to be able to produce flat $\left(\alpha_{\text {inj }} \approx 2.4\right)$ CR populations and they are believed to be the main CRp source of our galaxy (Schlickeiser 2002, and references therein). The reason for the steeper $\left(\alpha_{\mathrm{p}} \approx 2.7\right)$ galactic CRp spectrum is thought to be the momentum dependent escape probability from our Galaxy. Thus, the spectrum of CRp escaping from galaxies should be flat again $\left(\alpha_{\text {inj }} \approx 2.4\right)$. The spectrum injected into galaxy clusters could be even flatter, if termination shock waves of the galactic winds are able to reaccelerate them, as proposed by Völk et al. (1996).

The CRp population in galaxy clusters which is able to interact with the thermal gas and thus to produce observable signatures will be a mixture of contributions of the different sources, modified by acceleration and energy loss processes. In order not to rely too much on a specific physical picture, we discuss simplified models, which should be able to capture many typical situations.

\subsection{Spatial distribution of cosmic ray protons in cooling flow clusters}

In the following, we introduce three models for the spatial distribution of CRp within clusters of galaxies. The origin of the CRp population is not specified in the first two models, but the CRp may be accelerated by shock waves of cluster mergers, accretion shocks, or result from supernova driven galactic winds. In contrast to that we explore in the third model the diffusion process of CRp away from a central AGN. Since it is unclear how CRp are distributed spatially in detail, we investigate here three different scenarios which should serve as toy models. We pursue the philosophy of estimating physical parameters from observationally obtained electron density and temperature profiles by using simplified model assumptions for the CRp population. In doing so we try to minimize the dimensionality of parameter space as much as possible in order to track the main physical processes by means of analytically feasible methods and not to rely upon too many assumptions. 
Therefore the presented CRp profiles which are based on the assumption of spherical symmetry should not be interpreted as a precise estimate of the CRp population but rather as a plausible spherically averaged scenario.

\subsubsection{Isobaric model of CRp}

In this model we assume that the average kinetic CRp energy density $\varepsilon_{\mathrm{CRp}}(\boldsymbol{r})$ is a constant fraction of the thermal energy density $\varepsilon_{\mathrm{th}}(\boldsymbol{r})$ of the ICM

$\varepsilon_{\mathrm{CRp}}(\boldsymbol{r})=X_{\mathrm{CRp}} \varepsilon_{\mathrm{th}}(\boldsymbol{r})$.

This distribution might be maintained even in the case of a cooling flow cluster by mixing and ongoing turbulent CRp diffusion processes exerted by relativistic plasma bubbles rising in the gravitational potential of the cluster due to buoyant forces (Churazov et al. 2001, and references therein) which possibly leads to fractional pressure equilibrium with the thermal particle population.

\subsubsection{Adiabatic compression of CRp}

Here we assume the CRp population to be originally isobaric to the thermal population but to become adiabatically compressed during the formation of the cooling flow while it did not relax afterwards. The phase space volume stays constant during this transition and the momenta and volumes scale according to

$p_{\mathrm{p}} \rightarrow p_{\mathrm{p}}^{\prime}=\left(\frac{n_{\mathrm{e}}^{\prime}}{n_{\mathrm{e}}}\right)^{1 / 3} p_{\mathrm{p}}=C^{1 / 3} p_{\mathrm{p}}$,

$V_{\mathrm{p}} \rightarrow V_{\mathrm{p}}^{\prime}=\left(\frac{n_{\mathrm{e}}^{\prime}}{n_{\mathrm{e}}}\right)^{-1} V_{\mathrm{p}}=C^{-1} V_{\mathrm{p}}$

Here the compression factor $C=C(\boldsymbol{r})=\left(n_{\mathrm{e}}^{\prime} / n_{\mathrm{e}}\right)(\boldsymbol{r})$ has been introduced, which is larger than unity within cooling flows. Provided that the electrons have been in hydrostatic equilibrium during this transition, this implies $C(\boldsymbol{r})=T_{\text {cluster }} / T_{\mathrm{e}}^{\prime}(\boldsymbol{r})$, where $T_{\text {cluster }}$ denotes the electron temperature in the outer core region. This transformation implicitly assumes that the ratio of the CRp number densities before and after the adiabatic compression equals that of the electron population. If the differential number density distribution of the CRp population may be described by a power-law in momentum $p_{\mathrm{p}}$, then after adiabatic compression of $\mathrm{CRp}$ the functional shape of their distribution remains unchanged, however shifted according to

$f^{\prime}\left(\boldsymbol{r}^{\prime}, p^{\prime}\right)=\frac{\tilde{n}_{\mathrm{CRp}}^{\prime}\left(\boldsymbol{r}^{\prime}\right) c}{\mathrm{GeV}}\left(\frac{p^{\prime} c}{\mathrm{GeV}}\right)^{-\alpha_{\mathrm{p}}}$,

$\tilde{n}_{\mathrm{CRp}}^{\prime}\left(\boldsymbol{r}^{\prime}\right)=\tilde{n}_{\mathrm{CRp}}\left[\boldsymbol{r}^{\prime}(\boldsymbol{r})\right] C\left(\boldsymbol{r}^{\prime}\right)^{\left(\alpha_{\mathrm{p}}+2\right) / 3}$.

The normalization $\tilde{n}_{\mathrm{CRp}}(\boldsymbol{r})$ is chosen in such a way that the kinetic CRp energy density makes up a constant fraction of the thermal energy density prior to cooling flow formation and is described by a scaling parameter $X_{\mathrm{CRp}}$

$\varepsilon_{\mathrm{CRp}}(\boldsymbol{r})=X_{\mathrm{CRp}} \varepsilon_{\mathrm{th}}(\boldsymbol{r}) \rightarrow \varepsilon_{\mathrm{CRp}}^{\prime}\left(\boldsymbol{r}^{\prime}\right)=X_{\mathrm{CRp}}^{\prime}\left(\boldsymbol{r}^{\prime}\right) \varepsilon_{\mathrm{th}}\left(\boldsymbol{r}^{\prime}\right)$.

After adiabatic compression of $\mathrm{CRp}$ this scaling parameter has thus changed to

$X_{\mathrm{CRp}}^{\prime}(\boldsymbol{r})=C^{\left(\alpha_{\mathrm{p}}+2\right) / 3}(\boldsymbol{r}) X_{\mathrm{CRp}}$.
Since any hadronically induced emissivity scales with $X_{\mathrm{CRp}}^{\prime}$ we obtain the following relation,

$j^{\text {adiabatic }}(\boldsymbol{r})=C^{\left(\alpha_{\mathrm{p}}+2\right) / 3}(\boldsymbol{r}) j^{\text {isobaric }}(\boldsymbol{r})$.

\subsubsection{Diffusion of CRp away from a central $A G N$}

Many galaxy clusters - especially those with a cooling flow harbor a central galaxy, which often exhibits nuclear activity. The relativistic plasma bubbles produced by the AGN may contain relativistic protons, which can partly escape into the thermal ICM. Most of the CRp that have been injected into the cluster center are either diffusively transported into the surrounding ICM (as assumed by Colafrancesco \& Blasi 1998; Blasi 1999) or form relativistic bubbles which rise in the gravitational potential of the cluster due to buoyant forces (Churazov et al. 2001, and references therein). An argument in favor of a significant central CRp injection into the ICM is the much more efficient escape of CRp from the magnetic confinement of the radio plasma bubble during the very early stages due to the bubbles higher geometrical compactness and and expected stronger turbulence level (Enßlin 2003). In addition to this, any galactic wind from a central galaxy will also inject CRp into the cluster center. In order to treat these diffusion processes analytically one has to distinguish between clusters containing a cooling flow region or not. In the first case CRp diffusion will shape their emission profiles owing to the peaked cooling flow profiles while the emission strength in non-cooling flow clusters is mainly governed by the effective injection timescale.

Cooling flow clusters: The transport of CRp through the ICM is diffusive, with a diffusion coefficient $k(r, p)$ which in general may depend on momentum and position. For illustration we use

$\kappa(r, p)=\kappa_{0}(r)\left(\frac{p c}{\mathrm{GeV}}\right)^{\alpha_{\mathrm{diff}}}$,

with $\kappa_{0} \sim 10^{27 . .30} \mathrm{~cm}^{2} \mathrm{~s}^{-1}$ being plausible values. By using this ansatz, we ignore likely deviations of the diffusion coefficient from Eq. (56) in the mildly relativistic regime because these CRp are also not constrained by observations of their hadronic interactions. The coefficient $\alpha_{\text {diff }}$ describes the momentum dependence of the diffusion and is expected to be $\alpha_{\text {diff }} \approx \frac{1}{3}$ for active CRp diffusion in a Kolmogorov-like smallscale magnetic turbulence spectrum and $\alpha_{\text {diff }} \approx 0$ for passive advective transport in a turbulent flow. In the latter case $\kappa(p)=v_{\text {turb }} \lambda_{\text {turb }} / 3 \sim 10^{29} \mathrm{~cm}^{2} \mathrm{~s}^{-1}$, where $v_{\text {turb }} \sim 100 \mathrm{~km} \mathrm{~s}^{-1}$ and $\lambda_{\text {turb }} \sim 10 \mathrm{kpc}$ are the turbulent velocity and coherence length, respectively.

In a stationary situation, which is a valid approximation for timescales longer than the typical CRp diffusion timescale in the case of a stationary or short-term intermittent CRp source, the CRp distribution functions is given by

$f_{\mathrm{p}}\left(r, p_{\mathrm{p}}\right)=-\frac{Q(p)}{4 \pi} \int_{0}^{r} \frac{\mathrm{d} r^{\prime}}{\kappa\left(r^{\prime}, p\right) r^{\prime 2}}=\frac{Q_{\mathrm{p}}(p)}{4 \pi \kappa(p) r}$,

where $Q_{\mathrm{p}}(p)=\frac{Q_{\mathrm{p}, 0} c}{\mathrm{GeV}}\left(\frac{p_{\mathrm{p}} c}{\mathrm{GeV}}\right)^{-\alpha_{\mathrm{inj}}}$ 
Table 1. Parameters of electron density profiles $n_{\mathrm{e}}(r)$ of our cluster sample (central densities $n_{i}$ are subject to different formulae (66) and (67)). The cluster are ordered according to their property of being a cooling flow cluster (upper part) or a non-cooling flow cluster (lower part).

\begin{tabular}{|c|c|c|c|c|c|c|c|c|c|}
\hline Cluster & $z$ & $\begin{array}{c}n_{1} \\
{\left[h_{70}^{1 / 2} \mathrm{~cm}^{-3}\right]}\end{array}$ & $\begin{array}{c}r_{\mathrm{c}_{1}} \\
{\left[h_{70}^{-1} \mathrm{kpc}\right]}\end{array}$ & $\beta_{1}$ & $\begin{array}{c}n_{2} \\
{\left[h_{70}^{1 / 2} \mathrm{~cm}^{-3}\right]}\end{array}$ & $\begin{array}{c}r_{\mathrm{c}_{2}} \\
{\left[h_{70}^{-1} \mathrm{kpc}\right]}\end{array}$ & $\beta_{2}$ & Equation & References \\
\hline$\ldots \ldots \ldots \ldots$ & 0.0551 & $3.08 \times 10^{-2}$ & 45 & 0.662 & $3.87 \times 10^{-3}$ & 226 & 0.662 & (67) & (a), (b) \\
\hline A426 (Perseus) .... & 0.0179 & $4.6 \times 10^{-2}$ & 57 & 1.2 & $4.79 \times 10^{-3}$ & 200 & 0.58 & (66) & (c), (d) \\
\hline A2199 ........ & 0.0302 & $3.37 \times 10^{-2}$ & 29 & 0.663 & $7.17 \times 10^{-3}$ & 116 & 0.663 & (67) & (a), (b) \\
\hline A3526 (Centaurus) & 0.0114 & $8.05 \times 10^{-2}$ & 8.6 & 0.569 & $3.65 \times 10^{-3}$ & 99 & 0.569 & (67) & (a), (d) \\
\hline Ophiuchus ........ & 0.0280 & $1.71 \times 10^{-2}$ & 56 & 0.705 & $7.47 \times 10^{-3}$ & 190 & 0.705 & (67) & (a), (e) \\
\hline Triangulum Australis & 0.0510 & $7.31 \times 10^{-3}$ & 151 & 0.816 & $2.63 \times 10^{-3}$ & 444 & 0.816 & (67) & (a), (f) \\
\hline Virgo ............ & 0.0036 & $1.5 \times 10^{-1}$ & 1.6 & 0.42 & $1.3 \times 10^{-2}$ & 20 & 0.47 & (67) & (g), (h) \\
\hline A1656 (Coma) & 0.0231 & $3.4 \times 10^{-3}$ & 294 & 0.75 & & & & (66) & (i), (d) \\
\hline A2256 ...... & 0.0581 & $3.57 \times 10^{-3}$ & 347 & 0.828 & & & & (66) & (a), (d) \\
\hline A2319 & 0.0557 & $7.35 \times 10^{-3}$ & 152 & 0.536 & & & & (66) & (a), (d) \\
\hline A3571 & 0.0391 & $9.37 \times 10^{-3}$ & 124 & 0.61 & & & & (66) & (a), (d) \\
\hline
\end{tabular}

(a) Mohr et al. (1999), (b) Oegerle \& Hill (2001), (c) Churazov et al. (2003), (d) Struble \& Rood (1999), (e) Lahav et al. (1989), (f) McHardy et al. (1981), (g) Matsushita et al. (2002), (h) Ebeling et al. (1998), (i) Briel et al. (1992).

is the averaged CRp injection rate of the central source. We assume it to be a power-law in momentum with spectral in$\operatorname{dex} \alpha_{\text {inj }}$, which in general is not identical to the spectral index of the CRp population within radio plasma since the escape fraction is expected to depend on momentum (Enßlin 2003). For the last step in Eq. (57) we assume for simplicity the diffusion coefficient to be independent of position. Possible models of spatial distributions for the diffusion coefficient depend strongly on many unknown quantities such as the dominant diffusion mechanism (active diffusion versus passive advective transport), the velocity field, the turbulence scale, and the topology of the magnetic field, only to mention a few. Therefore we are unable to guess a realistic profile for $\kappa_{0}(r)$ without enlarging the accessible parameter space tremendously. However since we expect the diffusion coefficient not to change dramatically over the cooling flow scale and since the distribution function $f_{\mathrm{p}}\left(r, p_{\mathrm{p}}\right)$ is sufficiently steep in radius (Eq. (57)) our results should be approximately correct. The total CRp luminosity of the source can be estimated from Eq. (57) to be

$$
L_{\mathrm{CRp}}=\frac{m_{\mathrm{p}} c^{2} Q_{\mathrm{p}, 0}}{2\left(\alpha_{\mathrm{inj}}-1\right)}\left(\frac{m_{\mathrm{p}} c^{2}}{\mathrm{GeV}}\right)^{1-\alpha_{\mathrm{inj}}} \mathcal{B}\left(\frac{\alpha_{\mathrm{inj}}-2}{2}, \frac{3-\alpha_{\mathrm{inj}}}{2}\right) .
$$

Within our model, the CRp distribution function within the thermal ICM can be written as

$f_{\mathrm{p}}\left(r, p_{\mathrm{p}}\right)=\frac{\tilde{n}_{\mathrm{CRp}, 0} c}{\mathrm{GeV}}\left(\frac{r}{h_{70}^{-1} \mathrm{kpc}}\right)^{-1}\left(\frac{p_{\mathrm{p}} c}{\mathrm{GeV}}\right)^{-\alpha_{\mathrm{p}}}$,

where $\alpha_{\mathrm{p}}=\alpha_{\text {inj }}+\alpha_{\text {diff }}^{2}$.

\footnotetext{
2 This seems to be in contradiction to the identity of injection and equilibrium spectral index for a system without escape claimed in Sect. 3.1. Formally, we had to include particle escape from the galaxy cluster in order to be able to have a finite steady state solution of the diffusion problem, as given by Eq. (57). In the realistic case of a finite age of the system the stationary solution is only approximately valid in the center of the galaxy cluster. However, only there exists a
}

In order to obtain a realistic estimate for the diffusion volume to be considered, the relevant length scale needs to be taken into account. We define the characteristic scale $R_{\text {diff }}$ by calculating the second moment of the time-dependent distribution function of the first particles released by the source, yielding

$R_{\mathrm{diff}}=\sqrt{2 n_{\mathrm{dim}} t_{\mathrm{inj}} \kappa(p)} \simeq 80 h_{70}^{-1 / 2} \mathrm{kpc}\left(\frac{p c}{\mathrm{GeV}}\right)^{\alpha_{\mathrm{diff}} / 2}$,

where $n_{\mathrm{dim}}=3$ denotes the number of spatial dimensions. Here we assume a typical lifetime of $t_{\text {inj }}=3 h_{70}^{-1} \mathrm{Gyr}$ and $\kappa_{0} \simeq 10^{29} \mathrm{~cm}^{2} \mathrm{~s}^{-1}$. Beyond this scale $R_{\text {diff }}$ there can be a CRp population resulting from diffusion away from the central AGN which is however exponentially suppressed. Because the $\gamma$-ray luminosity resulting from hadronic CRp interactions scales as

$\mathcal{L}_{\gamma} \propto 4 \pi \int \mathrm{d} r r^{2} \tilde{n}_{\mathrm{CRp}}(r) n_{\mathrm{e}}(r) \propto \int \mathrm{d} r r^{1-3 \beta}$,

we always obtain centrally peaked $\gamma$-ray profiles, since the cooling radius is smaller than the diffusion scale, $r_{c_{1}}<R_{\mathrm{diff}}$, and $\beta>1 / 3$ within cooling flow regions (compare Table 1 ). Thus, the $\gamma$-ray luminosity is only weakly dependent on $R_{\text {diff }}$ as long as it reflects the correct order of magnitude.

In this work we constrain $\tilde{n}_{\mathrm{CRp}, 0}$ with the aid of $\gamma$-ray observations of galaxy clusters. From these constraints limits on the averaged CRp luminosity escaping from the radio plasma of the central galaxy can be derived using

$$
\begin{aligned}
\frac{L_{\mathrm{CRp}}}{\kappa_{0}}= & \frac{4 \pi m_{\mathrm{p}} c^{2} \tilde{n}_{\mathrm{CRp}, 0} h_{70}^{-1} \mathrm{kpc}}{2\left(\alpha_{\mathrm{inj}}-1\right)}\left(\frac{m_{\mathrm{p}} c^{2}}{\mathrm{GeV}}\right)^{1-\alpha_{\mathrm{inj}}} \\
& \times \mathcal{B}\left(\frac{\alpha_{\mathrm{inj}}-2}{2}, \frac{3-\alpha_{\mathrm{inj}}}{2}\right),
\end{aligned}
$$

sufficiently high target density to detect the CRp population. Therefore, although we use a poor description of the CRp profile on large-scales, the estimated $\gamma$-ray fluxes should be sufficiently accurate. 
Table 2. Parameters of temperature profiles $T_{\mathrm{e}}(r)$ of our cluster sample. The estimated $\gamma$-ray flux $\mathcal{F}_{\gamma \text {, est }}(>100 \mathrm{MeV})$ was calculated using the $\mathcal{F}_{\gamma}-F_{\mathrm{X}}$ scaling relation (Eq. (31)) with $\alpha_{\mathrm{p}}=2.3$ and bolometric X-ray fluxes from David et al. (1993). Note that $\mathcal{F}_{\gamma}$ scales linearly with $X_{\mathrm{CRp}}$ which was set to $X_{\mathrm{CRp}}=0.01 X_{0.01}$ in this table. The range for $\mathcal{F}_{\gamma \text {, est }}$ reflects the temperature spread in cooling flow clusters between the central temperature $T_{0}$ and the peripheral temperature $T_{1}$.

\begin{tabular}{|c|c|c|c|c|c|c|c|}
\hline Cluster & Experiment & $\begin{array}{c}k T_{0} \\
{[\mathrm{keV}]}\end{array}$ & $\begin{array}{c}k T_{1} \\
{[\mathrm{keV}]}\end{array}$ & $\begin{array}{c}r_{\text {temp }} \\
{\left[h_{70}^{-1} \mathrm{kpc}\right]}\end{array}$ & $\eta$ & $\begin{array}{c}\mathcal{F}_{\gamma, \text { est }}(>100 \mathrm{MeV}) \\
{\left[X_{0.01} 10^{-10} \mathrm{~cm}^{-2} \mathrm{~s}^{-1}\right]}\end{array}$ & References \\
\hline A $85 \ldots \ldots \ldots \ldots$ & BeppoSAX MECS & 5.5 & 9.0 & 312 & 2 & .. 2.3 & (a), (b) \\
\hline A426 (Perseus) .... & XMM-Newton MOS & 3.0 & 7.0 & 94 & 3 & $19.1 \quad \ldots 29.2$ & (c) \\
\hline A2199 ........... & Chandra ACIS & 1.6 & 4.3 & 21.5 & 1.8 & $\begin{array}{lll}1.3 & \ldots & 2.2\end{array}$ & (d), (e) \\
\hline A3526 (Centaurus) & ASCA GIS & 2.2 & 4.0 & 22 & 3 & $\begin{array}{lll}2.2 & \ldots & 2.9\end{array}$ & (f) \\
\hline Ophiuchus ........ & ASCA GIS & 12.8 & & & & 22.0 & (f) \\
\hline Triangulum Australis & ASCA GIS & 10.3 & & & & 4.8 & (f) \\
\hline Virgo $\ldots \ldots \ldots \ldots$ & XMM-Newton PN/MOS & 1.0 & 3.0 & 13.5 & 1 & ... 5.6 & $(\mathrm{~g})$ \\
\hline A1656 (Coma) ..... & XMM-Newton MOS & 8.3 & & & & 13.1 & (h) \\
\hline A2256 ...... & Chandra ACIS & 6.7 & & & & 2.3 & (i) \\
\hline A $2319 \ldots \ldots \ldots \ldots$ & ASCA GIS & 9.7 & & & & 5.4 & (f) \\
\hline A3571 $\ldots \ldots \ldots \ldots$ & ASCA GIS & 7.2 & & & & 4.5 & (f) \\
\hline
\end{tabular}

(a) Irwin \& Bregman (2000), (b) Lima Neto et al. (2001), (c) Churazov et al. (2003), (d) Voigt et al. (2002), (e) Johnstone et al. (2002), (f) White (2000), (g) Matsushita et al. (2002), (h) Arnaud et al. (2001), (i) Sun et al. (2002).

where we again ignored any possible low-energy spectral cutoff, since it can be included a posteriori with the help of Fig. 1. As a rough estimate we find numerically

$$
\begin{aligned}
L_{\mathrm{CRp}}= & L\left(\alpha_{\mathrm{inj}}\right) 10^{43} h_{70}^{-1 / 2} \mathrm{erg} \mathrm{s}^{-1} \\
& \times\left(\frac{\kappa_{0}}{10^{29} \mathrm{~cm}^{2} \mathrm{~s}^{-1}}\right)\left(\frac{\tilde{n}_{\mathrm{CRp}, 0}}{10^{-6} h_{70}^{1 / 2} \mathrm{~cm}^{-3}}\right),
\end{aligned}
$$

with $L\left(\alpha_{\text {inj }}\right)=6.1,2.2,1.6$, and 1.7 for $\alpha_{\text {inj }}=2.1,2.3,2.5$, and 2.7, respectively. In Sect 3.3.4 we analyze these constraints for our cluster sample in more detail.

Non-cooling flow clusters: In transforming the above considerations on diffusion length scales to the case of non-cooling flow clusters we point out the following differences: In noncooling flow clusters the core radius is normally larger than the diffusion scale, $r_{\mathrm{c}}>R_{\mathrm{diff}}$, over which the electron density varies only slightly. Thus, a stationary solution to the diffusion equation is not applicable in the case of a flat target profile. It follows that the volume integrated $\gamma$-ray spectrum does not depend on the diffusion coefficient but only on the injection time $t_{\text {inj }}$ of CRp into the ICM of the cluster core. We therefore adopt a modification to the diffusion model for noncooling flow clusters. The averaged CRp luminosity of the central galaxy reads in this context

$$
\begin{aligned}
L_{\mathrm{CRp}}= & \frac{\tilde{N}_{\mathrm{CRp}}}{t_{\mathrm{inj}}} \frac{m_{\mathrm{p}} c^{2}}{2\left(\alpha_{\mathrm{inj}}-1\right)}\left(\frac{m_{\mathrm{p}} c^{2}}{\mathrm{GeV}}\right)^{1-\alpha_{\mathrm{inj}}} \\
& \times \mathcal{B}\left(\frac{\alpha_{\mathrm{inj}}-2}{2}, \frac{3-\alpha_{\mathrm{inj}}}{2}\right) .
\end{aligned}
$$

Here $\tilde{N}_{\mathrm{CRp}}$ denotes the integrated number of CRp being injected into the ICM of the cluster and $\alpha_{\text {inj }}=\alpha_{\mathrm{p}}$, because there is no diffusion induced spectral steepening simply due to the fact that the even more energetic CRp which are still significantly contributing to the $\gamma$-ray flux in the EGRET energy band are not able to leave the central core region within a reasonable timescale.

\subsection{Constraining the population of $C R p$ by the integrated flux of $\gamma$-rays in different clusters}

\subsubsection{Cluster sample}

Applying the $\mathcal{F}_{\gamma}-F_{\mathrm{X}}$ scaling relation (Eq. (31)) and taking bolometric X-ray fluxes from David et al. (1993) while fixing $X_{\mathrm{CRp}}=0.01 X_{0.01}$ and $\alpha_{\mathrm{p}}=2.3$ we estimated $\gamma$-ray fluxes $\mathcal{F}_{\gamma \text {, est }}(>100 \mathrm{MeV})$ for the spectral sensitivity of EGRET in order to choose our cluster sample (see Table 2). Inferred values for the estimated $\gamma$-ray flux $\mathcal{F}_{\gamma \text {, est }}$ by means of the $\mathcal{F}_{\gamma}-F_{\mathrm{X}}$ scaling relation sensitively depend on the bolometric X-ray luminosity of the particular cluster such that values for $\mathcal{F}_{\gamma \text {, est }}$ in Table 2 represent a rough estimate. A detailed modeling using density and temperature profiles will be described later on in Sect. 3.3.2 in order to obtain upper limits on the CRp population. By comparing $\gamma$-ray fluxes $\mathcal{F}_{\gamma}$ obtained from these two different methods we recognized an inconsistency for the Virgo and Centaurus cluster: this discrepancy is explained by a too small aperture of the X-ray experiments analyzed by David et al. (1993) giving rise to an underestimation of the X-ray flux of these two nearest clusters in our sample $\left(z_{\text {Virgo }}=0.0036\right.$ and $z_{\text {Centaurus }}=0.0114$ ) and therefore an underestimate of $\mathcal{F}_{\gamma \text {, est }}$ for these two clusters. Moreover, we noticed a systematic discrepancy of the order of $50 \%$ between the different methods in cooling flow clusters which is due to an insufficient accounting for the radial temperature variation in Eq. (31).

Parameters of electron density profiles $n_{\mathrm{e}}(r)$ of our cluster sample are given in Table 1 where the clusters are ordered according totheir property of containing a cooling flow 
Table 3. Upper limits on the CRp scaling parameter $X_{\mathrm{CRp}}$ by comparing the integrated flux above $100 \mathrm{MeV}$ to EGRET upper limits assuming a $\gamma$-ray spectral index in Dermer's model $\alpha_{\gamma}=\alpha_{\mathrm{p}}$. The spatial distribution of CRp is given by the isobaric and the adiabatic model of CRp, respectively (see Sects. 3.2.1 and 3.2.2).

\begin{tabular}{|c|c|c|c|c|c|c|c|c|c|}
\hline \multirow[b]{2}{*}{ Cluster } & \multirow{2}{*}{$\begin{array}{l}\mathcal{F}_{\gamma}(>100 \mathrm{MeV}) \\
{\left[10^{-8} \mathrm{~cm}^{-2} \mathrm{~s}^{-1}\right]}\end{array}$} & \multicolumn{4}{|c|}{$X_{\mathrm{CRp}}^{\text {isobaric }}$} & \multicolumn{4}{|c|}{$X_{\mathrm{CRp}}^{\text {adiabatic }}$} \\
\hline & & $\alpha_{\mathrm{p}}=2.1$ & $\alpha_{\mathrm{p}}=2.3$ & $\alpha_{\mathrm{p}}=2.5$ & $\alpha_{\mathrm{p}}=2.7$ & $\alpha_{\mathrm{p}}=2.1$ & $\alpha_{\mathrm{p}}=2.3$ & $\alpha_{\mathrm{p}}=2.5$ & $\alpha_{\mathrm{p}}=2.7$ \\
\hline A85 ............. & $<6.32$ & 3.53 & 1.97 & 2.09 & 3.11 & 2.58 & 1.41 & 1.48 & 2.16 \\
\hline A426 (Perseus) .... & $<3.72$ & 0.14 & 0.08 & 0.08 & 0.13 & 0.12 & 0.06 & 0.07 & 0.10 \\
\hline A2199 ......... & $<9.27$ & 6.14 & 3.42 & 3.64 & 5.42 & 5.74 & 3.18 & 3.38 & 5.00 \\
\hline A3526 (Centaurus) & $<5.31$ & 1.54 & 0.86 & 0.91 & 1.36 & 1.45 & 0.80 & 0.85 & 1.26 \\
\hline Ophiuchus ......... & $<5.00$ & 0.30 & 0.17 & 0.18 & 0.26 & & & & \\
\hline Triangulum Australis & $<8.13$ & 1.93 & 1.07 & 1.14 & 1.70 & & & & \\
\hline Virgo ............ & $<2.18$ & 0.18 & 0.10 & 0.11 & 0.16 & 0.16 & 0.09 & 0.09 & 0.14 \\
\hline A1656 (Coma) ..... & $<3.81$ & 0.45 & 0.25 & 0.27 & 0.40 & & & & \\
\hline A2256 .......... & $<4.28$ & 3.15 & 1.75 & 1.87 & 2.78 & & & & \\
\hline A2319. & $<3.79$ & 0.86 & 0.48 & 0.51 & 0.76 & & & & \\
\hline$\ldots \ldots \ldots$ & $<6.34$ & 1.85 & 1.03 & 1.09 & 1.63 & & & & \\
\hline
\end{tabular}

(upper part) or not (lower part). Note that the parameters are subject to different formulae (66) and (67),

$$
\begin{aligned}
& n_{\mathrm{e}}(r)=\sum_{i=1}^{2} n_{i}\left(1+\frac{r^{2}}{r_{\mathrm{c}_{i}}^{2}}\right)^{-3 \beta / 2}, \\
& n_{\mathrm{e}}(r)=\left[\frac{\tilde{\Lambda}\left[T_{\mathrm{e}}(0)\right]}{\tilde{\Lambda}\left[T_{\mathrm{e}}(r)\right]} \times \sum_{i=1}^{2} n_{i}^{2}\left(1+\frac{r^{2}}{r_{\mathrm{c}_{i}}^{2}}\right)^{-3 \beta}\right]^{1 / 2} .
\end{aligned}
$$

The last Eq. (67) follows from deprojection of X-ray surface brightness profiles which are represented by double $\beta$ models. The derivation of this deprojection is given in Appendix A. For simplicity and consistency with the X-ray surface brightness profiles given in Mohr et al. (1999) we ignored the weak dependency on $T_{\mathrm{e}}(r)$ in Eq. (67).

In order to model the temperature profiles $T_{\mathrm{e}}(r)$ for our cooling flow cluster sample we applied the universal temperature profile for relaxed clusters proposed by Allen et al. (2001) to data taken from the literature,

$$
T_{\mathrm{e}}(r)=T_{0}+\left(T_{1}-T_{0}\right)\left[1+\left(\frac{r}{r_{\text {temp }}}\right)^{-\eta}\right]^{-1} .
$$

This equation matches the temperature profile well up to radii of $\sim 0.3 r_{\text {vir }}$, which is sufficient for our purposes since we are especially interested in the core region of clusters. The parameters of the temperature profile for particular cluster are given in Table 2.

\subsubsection{Simulated $\gamma$-ray flux normalized by EGRET limits: The case of Perseus cluster}

The volume integrated omnidirectional differential $\gamma$-ray source function $Q_{\gamma}\left(E_{\gamma}\right)$ can be obtained by integrating Eq. (19). We integrated the volume out to a radius of $3 h_{70}^{-1} \mathrm{Mpc}$ which corresponds to the characteristic distance where the simple $\beta$-model of electron densities breaks down due to accretion shocks in clusters. The integration kernel $q_{\gamma}\left(\boldsymbol{r}, E_{\gamma}\right)$ scales linearly with $\tilde{n}_{\mathrm{CRp}}(\boldsymbol{r})$ (as shown in Eq. (19)) which is obtained by solving Eqs. (7) and (10). By comparing the integrated $\gamma$-ray flux above $100 \mathrm{MeV}, \mathcal{F}_{\gamma}(>100 \mathrm{MeV})$, to EGRET upper limits (see Reimer et al. 2003), we constrain the CRp scaling parameter $X_{\mathrm{CRp}}$. The inferred value for $X_{\mathrm{CRp}}$ in the Perseus cluster normalizes the differential $\gamma$-ray flux

$\frac{\mathrm{d} \mathcal{F}_{\gamma}}{\mathrm{d} E_{\gamma}} \equiv \frac{Q_{\gamma}\left(E_{\gamma}\right)}{4 \pi D^{2}}$

in Fig. 3. The $\pi^{0}$-meson decay induced distinct spectral signature resulting in the peak at a $\gamma$-ray energy of $m_{\pi^{0}} c^{2} / 2 \simeq$ 67.5 MeV can be clearly seen.

Figure 3 shows also upper limits on the differential $\gamma$-ray flux owing to IC emission of hadronically originating CRe represented by power-laws. The IC spectra are computed by means of Eq. (43) for different spectral indices $\alpha_{\mathrm{p}}$ and zero magnetic field. Non-zero magnetic fields can be included since the IC spectra scale according to $B_{\mathrm{CMB}}^{2} /\left(B(\boldsymbol{r})^{2}+B_{\mathrm{CMB}}^{2}\right.$ ) (see Eq. (36)) which results in a lower normalization.

\subsubsection{Results on the scaling parameter $X_{\mathrm{CRp}}$ using $\gamma$-ray observations in different clusters}

By employing the technique described in Sect. 3.3.2 we constrained the CRp scaling parameter $X_{\mathrm{CRp}}$ using EGRET upper limits of the $\gamma$-ray flux by Reimer et al. (2003). As described in that section, we infer the $\gamma$-ray flux of this clusters originating from within a sphere of radius $3 h_{70}^{-1}$ Mpc. Owing to the vicinity of the Virgo cluster this maximum radius subtends an angle on the sky which is larger than the width of the point spread function of the EGRET instrument $\left(\theta_{\max }=\right.$ $5.8^{\circ}\left[E_{\gamma} /(100 \mathrm{MeV})\right]^{-0.534}$, Reimer et al. 2003). Thus in the case of Virgo we use this smaller integration volume. Table 3 shows constraints for $X_{\mathrm{CRp}}$ using the isobaric and the adiabatic model of CRp described in Sect. 3.2. Because in the adiabatic model the CRp scaling parameter $X_{\mathrm{CRp}}$ is a function of radius, the value $X_{\mathrm{CRp}}^{\text {adiabatic }}$ given in Table 3 refers to the unprimed quantity in Eq. (54) which reflects the outer core region of the cluster. For clusters like Perseus (A 426), Virgo, Ophiuchus, 
Table 4. Cooling flow clusters: upper limits on the CRp density parameter $\tilde{n}_{\mathrm{CRp}, 0}$ and average CRp luminosity $L_{\mathrm{CRp}}$ of the central active galaxy by comparing the integrated flux above $100 \mathrm{MeV}$ to EGRET upper limits assuming a $\gamma$-ray spectral index in Dermer's model $\alpha_{\gamma}=\alpha_{\mathrm{p}}$. The spatial distribution of CRp is is calculated according to the diffusion model of CRp away from a central AGN assuming $\alpha_{\mathrm{p}}=\alpha_{\text {inj }}+\alpha_{\text {diff }}$, where $\alpha_{\text {diff }}=1 / 3$. Non-cooling flow clusters: upper limits on the CRp number parameter $\tilde{N}_{\text {CRp }}$ and average CRp luminosity $L_{\text {CRp }}$ without any diffusion induced spectral steepening, i.e. $\alpha_{\mathrm{p}}=\alpha_{\text {inj. }}$. Note that $L_{\mathrm{CRp}}$ scales in the case of cooling flow clusters with the diffusion coefficient $\kappa_{0}$ while it only depends on the CRp injection time $t_{\text {inj }}$ for non-cooling flow clusters (see Sect. 3.2.3).

\begin{tabular}{|c|c|c|c|c|c|c|c|c|}
\hline \multirow[b]{2}{*}{ CF Cluster } & \multicolumn{4}{|c|}{$\tilde{n}_{\mathrm{CRp}, 0}\left[h_{70}^{1 / 2} \mathrm{~cm}^{-3}\right]$} & \multicolumn{4}{|c|}{$L_{\mathrm{CRp}}\left[h_{70}^{-1 / 2} \operatorname{erg~s}^{-1}\left(\frac{\kappa_{0}}{10^{29} \mathrm{~cm}^{2} \mathrm{~s}^{-1}}\right)\right]$} \\
\hline & $\alpha_{\mathrm{p}}=2.4$ & $\alpha_{\mathrm{p}}=2.5$ & $\alpha_{\mathrm{p}}=2.7$ & $\alpha_{\mathrm{p}}=2.9$ & $\alpha_{\mathrm{p}}=2.4$ & $\alpha_{\mathrm{p}}=2.5$ & $\alpha_{\mathrm{p}}=2.7$ & $\alpha_{\mathrm{p}}=2.9$ \\
\hline A85 & $6.0 \times 10^{-5}$ & $7.2 \times 10^{-5}$ & $9.9 \times 10^{-5}$ & $1.3 \times 10^{-4}$ & $5.5 \times 10^{45}$ & $2.7 \times 10^{45}$ & $1.9 \times 10^{45}$ & $2.0 \times 10^{45}$ \\
\hline A426 (Perseus) & $2.4 \times 10^{-6}$ & $2.9 \times 10^{-6}$ & $3.9 \times 10^{-6}$ & $5.2 \times 10^{-6}$ & $2.2 \times 10^{44}$ & $1.1 \times 10^{44}$ & $7.4 \times 10^{43}$ & $8.1 \times 10^{43}$ \\
\hline A2199 $\ldots \ldots \ldots$ & $3.2 \times 10^{-5}$ & $3.8 \times 10^{-5}$ & $5.3 \times 10^{-5}$ & $7.0 \times 10^{-5}$ & $3.0 \times 10^{45}$ & $1.4 \times 10^{45}$ & $9.9 \times 10^{44}$ & $1.1 \times 10^{45}$ \\
\hline A3526 (Centaurus) & $4.3 \times 10^{-6}$ & $5.1 \times 10^{-6}$ & $7.1 \times 10^{-6}$ & $9.4 \times 10^{-6}$ & $3.9 \times 10^{44}$ & $1.9 \times 10^{44}$ & $1.3 \times 10^{44}$ & $1.5 \times 10^{44}$ \\
\hline Ophiuchus ....... & $1.5 \times 10^{-5}$ & $1.8 \times 10^{-5}$ & $2.5 \times 10^{-5}$ & $3.3 \times 10^{-5}$ & $1.4 \times 10^{45}$ & $6.6 \times 10^{44}$ & $4.6 \times 10^{44}$ & $5.1 \times 10^{44}$ \\
\hline Triangulum Australis & $1.4 \times 10^{-4}$ & $1.7 \times 10^{-4}$ & $2.3 \times 10^{-4}$ & $3.1 \times 10^{-4}$ & $1.3 \times 10^{46}$ & $6.2 \times 10^{45}$ & $4.3 \times 10^{45}$ & $4.8 \times 10^{45}$ \\
\hline \multirow[t]{2}{*}{ Virgo...$\ldots \ldots \ldots$} & $2.5 \times 10^{-7}$ & $3.1 \times 10^{-7}$ & $4.2 \times 10^{-7}$ & $5.6 \times 10^{-7}$ & $2.4 \times 10^{43}$ & $1.1 \times 10^{43}$ & $7.9 \times 10^{42}$ & $8.7 \times 10^{42}$ \\
\hline & \multicolumn{4}{|c|}{$\tilde{N}_{\mathrm{CRp}}\left[h_{70}^{1 / 2}\right]$} & \multicolumn{4}{|c|}{$L_{\mathrm{CRp}}\left[h_{70}^{3 / 2} \operatorname{erg~s}^{-1}\left(\frac{t_{\text {inj }}}{3 \text { Gyr }}\right)^{-1}\right]$} \\
\hline NCF Cluster & $\alpha_{\mathrm{p}}=2.1$ & $\alpha_{\mathrm{p}}=2.3$ & $\alpha_{\mathrm{p}}=2.5$ & $\alpha_{\mathrm{p}}=2.7$ & $\alpha_{\mathrm{p}}=2.1$ & $\alpha_{\mathrm{p}}=2.3$ & $\alpha_{\mathrm{p}}=2.5$ & $\alpha_{\mathrm{p}}=2.7$ \\
\hline A1656 (Coma) & $1.6 \times 10^{64}$ & $2.5 \times 10^{64}$ & $3.7 \times 10^{64}$ & $5.1 \times 10^{64}$ & $2.7 \times 10^{45}$ & $1.5 \times 10^{45}$ & $1.6 \times 10^{45}$ & $2.4 \times 10^{45}$ \\
\hline A $2256 \ldots \ldots$ & $1.1 \times 10^{65}$ & $1.8 \times 10^{65}$ & $2.6 \times 10^{65}$ & $3.6 \times 10^{65}$ & $1.9 \times 10^{46}$ & $1.1 \times 10^{46}$ & $1.1 \times 10^{46}$ & $1.7 \times 10^{46}$ \\
\hline A2319 & $4.5 \times 10^{64}$ & $7.0 \times 10^{64}$ & $1.0 \times 10^{65}$ & $1.4 \times 10^{65}$ & $7.5 \times 10^{45}$ & $4.2 \times 10^{45}$ & $4.4 \times 10^{45}$ & $6.6 \times 10^{45}$ \\
\hline A3571 $\ldots \ldots \ldots \ldots$ & $2.8 \times 10^{64}$ & $4.4 \times 10^{64}$ & $6.5 \times 10^{64}$ & $9.0 \times 10^{64}$ & $4.7 \times 10^{45}$ & $2.6 \times 10^{45}$ & $2.8 \times 10^{45}$ & $4.2 \times 10^{45}$ \\
\hline
\end{tabular}

and Coma (A 1656) we can obtain quite tight constraints on the population of CRp.

\subsubsection{Results on $L_{C R p}$ in the $A G N$-diffusion model}

The procedure of inferring constraints on CRp diffusing away from a central source is mostly sensitive to the CRp population of the central cooling flow region rather than the shock region in the outer parts of the cluster. In order to constrain the CRp density parameter $\tilde{n}_{\mathrm{CRp}, 0}$ and averaged CRp luminosity $L_{\mathrm{CRp}}$ of the central active galaxy in our AGN-diffusion model of cooling flow clusters we have to calculate the volume integrated omnidirectional differential $\gamma$-ray source function $Q_{\gamma}\left(E_{\gamma}\right)$ (see Eq. (2)). The integration kernel $q_{\gamma}\left(E_{\gamma}\right)$ is proportional to $\tilde{n}_{\mathrm{CRp}}(\boldsymbol{r})$ (Eq. (19)) which is obtained by solving Eqs. (6) and (60). By comparing the integrated $\gamma$-ray flux above $100 \mathrm{MeV}$ to EGRET upper limits (see Reimer et al. 2003), we constrain the CRp density parameter $\tilde{n}_{\mathrm{CRp}, 0}$. In the case of noncooling flow clusters we constrain the averaged CRp luminosity $L_{\mathrm{CRp}}$ with the aid of the integrated CRp number parameter $\tilde{N}_{\text {CRp }}$, yielding an indirect measure of a combination of the CRp escape fraction from the radio plasma of the central galaxy and the averaged CRp luminosity of this source.

Upper limits on the CRp density parameter $\tilde{n}_{\mathrm{CRp}, 0}$, number parameter of CRp $\tilde{N}_{\mathrm{CRp}}$, and averaged CRp luminosity $L_{\mathrm{CRp}}$ of the central active galaxy (by means of Eq. (63)) are presented in Table 4. This shows that within this conceptually simple model we are able to put constraints on the averaged CRp luminosity $L_{\mathrm{CRp}}$. The limits which are strongest in the case of M87 in the Virgo cluster represent conservative bounds since we choose the active CRp diffusion scenario resulting in spectral steepening of the CRp population. We obtain even tighter limits when assuming a passive advective transport of the CRp in a turbulent flow in which case we infer

$L_{\mathrm{CRp}}=L\left(\alpha_{\mathrm{p}}\right) 10^{42} h_{70}^{-1 / 2} \operatorname{erg~s}^{-1}\left(\frac{\kappa_{0}}{10^{29} \mathrm{~cm}^{2} \mathrm{~s}^{-1}}\right)$

with $L\left(\alpha_{\mathrm{p}}\right)=4.5,4.8,7.2$, and 20.9 for $\alpha_{\mathrm{p}}=2.4,2.5,2.7$, and 2.9, respectively. These values are slightly smaller than instantaneous jet power estimates of M87 being of the order of $L_{\text {jet }} \simeq 10^{43} \mathrm{erg} \mathrm{s}^{-1}$ (Bicknell \& Begelman 1996; Young et al. 2002). In general, this demonstrates the ability of future high resolution $\gamma$-ray observations to constrain the energy fraction of CRp escaping from the radio plasma.

\subsection{Radio emissivity of secondary electrons: The case of the radio mini-halo in Perseus}

In contrast to $\gamma$-rays induced by hadronic CRp interactions whose spectral shape and normalization is only governed by the spectral index $\alpha_{\mathrm{p}}$ as free parameter, the resulting radio emission from secondary electrons also depends on the morphology and strength of the magnetic field $B(\boldsymbol{r})$. Because only a subsample of cooling flow clusters contain radio mini-halos which are not outshined by the central AGN we decided to concentrate on the Perseus cluster. It has the fortunate property that the radio emission due to the central galaxy NGC 1275 is spatially resolved and can be separated from the diffuse emission due to the radio-mini halo. 


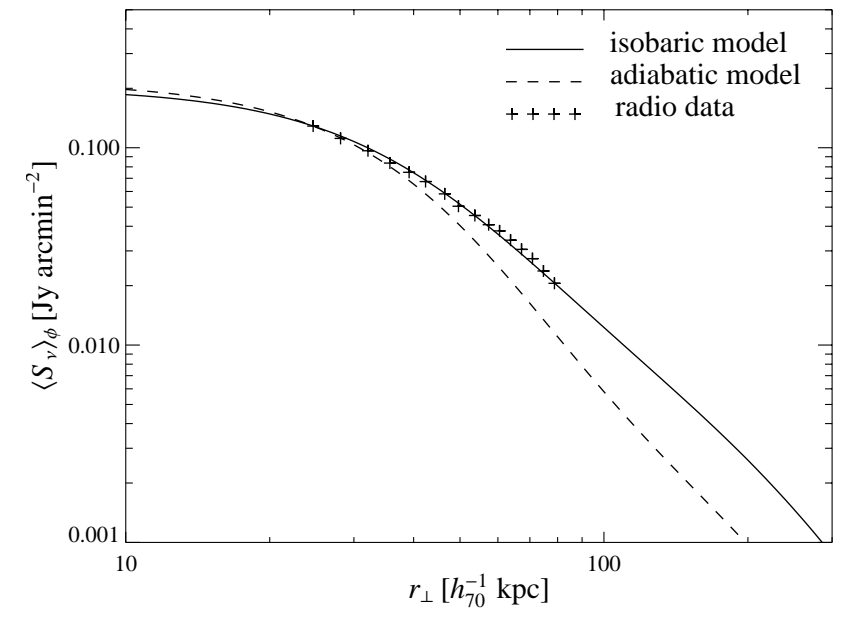

Fig. 4. The radial distribution of radio brightness as a function of impact parameter $r_{\perp}$. Shown are the CRp adiabatic and isobaric model for model parameters $B_{0}=10 \mu \mathrm{G}, \alpha_{B}=0.5$, and $\alpha_{\mathrm{p}}=2.3$ (details are described in the text) as well as the azimuthally averaged radio brightness profile of the the Perseus radio-mini halo (data was taken from Pedlar et al. 1990). The normalization of the radio brightness depends on the assumed scaling between CRp and thermal energy density. We fix this scaling parameter $X_{\mathrm{CRp}}$ by comparing the simulated radio brightness to the measured data at $24.65 h_{70}^{-1} \mathrm{kpc}$.

\subsubsection{Intracluster magnetic fields}

Magnetic fields in galaxy clusters seem to be on the level of $\sim \mu \mathrm{G}$. Indirect estimates of magnetic field strength assuming equipartition of energy density of the fields and that of a radio synchrotron emitting relativistic electron population give low field strengths of $\sim 0.1 \mu \mathrm{G}$. Also lower limits on the field strength of a comparable level can be derived using the measurements or upper limits on IC scattered CMB photons in the hard X-ray band (Rephaeli et al. 1994; Fusco-Femiano et al. 1999; Enßlin et al. 1999). Conversely, Faraday rotation measurements indicate magnetic fields strengths of several $\mu \mathrm{G}$ in typical galaxy clusters and a few $10 \mu \mathrm{G}$ in cooling flow regions of clusters (Carilli \& Taylor 2002, for a review). Faraday rotation based measurements of the field strength depend on estimating the magnetic autocorrelation length from fluctuations in the Faraday rotation maps. Although the formerly used methods to estimate this length-scale seem to be questionable (Enßlin \& Vogt 2003) a refined analysis gives comparable results for the magnetic field strengths (Vogt \& Enßlin 2003, in preparation).

\subsubsection{Comparison of the morphology of radio emissivity from secondary electrons}

The radio data was taken from Pedlar et al. (1990) where we neglected the innermost data points because of enhanced contribution to radio brightness of the radio jet of NGC 1275 and the outermost data points due to the limited sensitivity on the larger scales of the specific VLA configuration likely leading to an artificial decline in the radio surface brightness. The values for the azimuthally averaged radio surface brightness were converted assuming a two-dimensional Gaussian beam which
Table 5. Upper limits on the CRp scaling parameter $X_{\mathrm{CRp}}$ inferred from radio brightness profiles of the radio mini-halo of Perseus cluster for different values of $B_{0}, \alpha_{B}$, and $\alpha_{\mathrm{p}}$.

\begin{tabular}{cccccc}
\hline \hline Model & $\alpha_{\mathrm{p}}$ & $B_{0}[\mu \mathrm{G}]$ & $\alpha_{B}$ & $X_{\mathrm{CRp}}^{\text {isobaric }}$ & $X_{\mathrm{CRp}}^{\text {adiabatic }}$ \\
\hline 1 & 2.3 & 10 & 0.5 & 0.016 & 0.006 \\
2 & 2.1 & 10 & 0.5 & 0.014 & 0.005 \\
3 & 2.5 & 10 & 0.5 & 0.033 & 0.011 \\
4 & 2.7 & 10 & 0.5 & 0.096 & 0.031 \\
5 & 2.3 & 5 & 0.5 & 0.027 & 0.009 \\
6 & 2.3 & 20 & 0.5 & 0.012 & 0.004 \\
7 & 2.3 & 10 & 0.7 & 0.017 & 0.006 \\
8 & 2.3 & 10 & 0.9 & 0.019 & 0.006 \\
\hline
\end{tabular}

leads to a beam area $A_{\text {beam }}=\pi(4 \ln 2)^{-1} F W H M_{x} F W H M_{y}$. Figure 4 shows the radial distribution of radio brightness $S_{v}\left(r_{\perp}\right)$ as a function of impact parameter $r_{\perp}$ obtained by means of Eq. (42) in comparison to the radio data. The CRp adiabatic and isobaric model being described in Sect. 3.2 are both shown using model parameters of $\alpha_{\mathrm{p}}=2.3, B_{0}=10 \mu \mathrm{G}$, and $\alpha_{B}=0.5$, where the latter two parameters refer to Eq. (41). The normalization of the radio brightness depends on the assumed scaling between CRp and thermal energy density. We fix this scaling parameter $X_{\mathrm{CRp}}$ by comparing the simulated radio brightness to the measured data at $24.65 h_{70}^{-1} \mathrm{kpc}$. There is an excellent morphological concordance of the isobaric model of CRp and the radio data for the radio-mini halo of the Perseus cluster. Since the required values of $X_{\mathrm{CRp}}$ are plausible ( 0.01-0.1, see Sect. 3.4.3), the hadronic secondary CRe model is a very attractive explanation for the observed radio mini-halos in cooling flow clusters.

\subsubsection{Results on the scaling parameter $X_{\mathrm{CRp}}$ using radio observations in different models}

By comparing the simulated radio brightness to the measured radio data at $24.65 h_{70}^{-1} \mathrm{kpc}$ which is the innermost azimuthally averaged data point not being outshined by the radio galaxy cocoon of NGC 1275 we determine the CRp scaling parameter $X_{\mathrm{CRp}}$. Taking this point of reference yields more conservative upper limits for $X_{\mathrm{CRp}}$ instead of normalizing by the integrated radio surface brightness especially in the case of poorer morphological matches. The inferred values for $X_{\mathrm{CRp}}$ in Table 5 are shown for different combinations of $B_{0}, \alpha_{B}$, and $\alpha_{\mathrm{p}}$.

Deduced values of this scaling parameter $X_{\mathrm{CRp}}$ which are obtained by considering only pion decay induced secondary electrons resulting from hadronic CRp interactions in the ICM reflect upper limits because there are also other mechanisms in galaxy clusters leading to relativistic populations of electrons (see Sect. 1). By analyzing the variations of our model parameters in Table 5 we conclude a weak dependence of $X_{\mathrm{CRp}}$ on $\alpha_{B}$ while the magnetic field strength at the cluster center $B_{0}$ and the CRp spectral index $\alpha_{\mathrm{p}}$ show a stronger influence on $X_{\mathrm{CRp}}$. The spectral parameter of the magnetic field $\alpha_{B}$ impacts mostly on the radial extensions of the radio brightness profiles while 


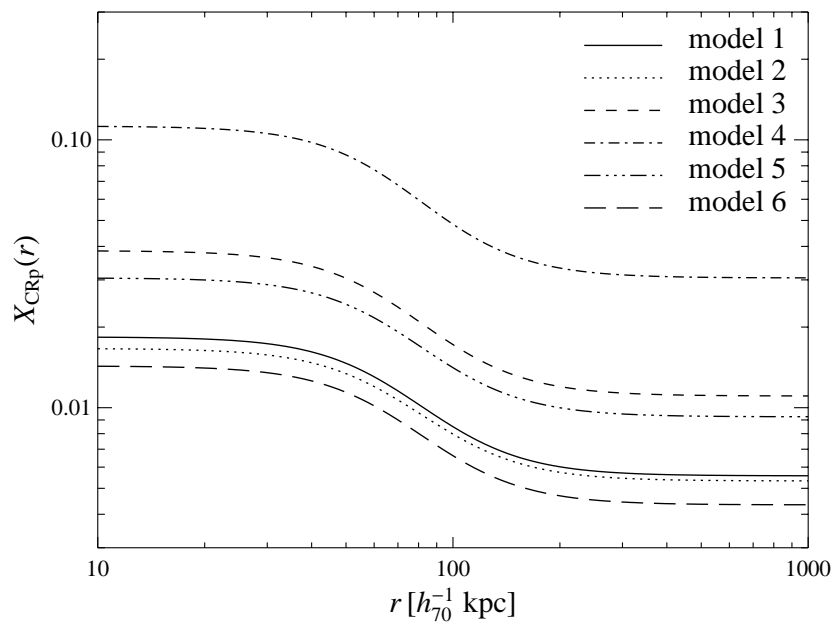

Fig. 5. The deprojected scaling parameter $X_{\mathrm{CRp}}(r)$ between CRp and thermal energy density in the adiabatic model applied to the miniradio halo of Perseus and presented for models which are defined in Table 5.

the CRp scaling parameter reflects a degeneracy with respect to $B_{0}$ and $\alpha_{\mathrm{p}}$.

Figure 5 shows the scaling parameter $X_{\mathrm{CRp}}(r)$ as a function of radius $r$ between CRp and thermal energy density in the adiabatic model according to Eq. (54) for models defined in Table 5. The enhancement of CRp relative to the thermal energy density owing to adiabatic compression of the CRp population during the formation of the cooling flow can be clearly seen.

\subsection{Constraints derived from the radio halo of Coma}

\subsubsection{Parameter study of the hadronic scenario}

We also applied this formalism of synchrotron radiation emitted by secondary electrons as presented in Sect. 2.4 to the radial distribution of radio brightness in the radio halo of the Coma cluster using radio data at $1.4 \mathrm{GHz}$ by Deiss et al. (1997). Assuming the CRp population to be distributed according to the isobaric model, the spatial radio brightness profile obtained by this secondary electron model declines too fast with increasing impact parameter $r_{\perp}$ in order to account for the observed extended radio halo of Coma. To check whether this shortfall of the theoretical model represents a serious problem for the hadronic model of radio synchrotron emission we are asking in turn for the necessary radial variation of the CRp scaling parameter $X_{\mathrm{CRp}}(r)$ that is able to explain the observed radio halo. Deprojecting the azimuthally averaged observed radio surface brightness profile which is described by a $\beta$-model yields (as laid down in Appendix A)

$j_{v}(r)=\frac{S_{0}}{2 \pi r_{\mathrm{c}}} \frac{6 \beta-1}{\left(1+r^{2} / r_{\mathrm{c}}^{2}\right)^{3 \beta}} \mathcal{B}\left(\frac{1}{2}, 3 \beta\right)$,

where $S_{0}=1.1 \mathrm{mJy} \operatorname{arcmin}^{-2}, r_{\mathrm{c}}=450 h_{70}^{-1} \mathrm{kpc}$, and $\beta=0.78$. By comparing the observed to the theoretically expected radio emissivity at each radius we infer the ratio of CRp-tothermal energy density $X_{\mathrm{CRp}}(r)$. Figure 6 shows a comparison

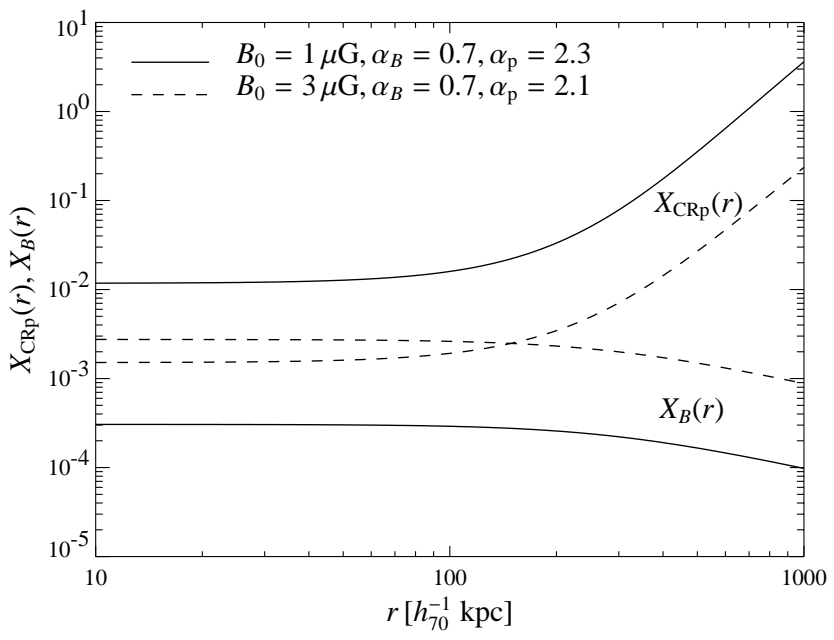

Fig. 6. The deprojected CRp scaling parameter $X_{\mathrm{CRp}}(r)$ required to account for the observed radio halo in Coma within the framework of the hadronic scenario. The rising curves with increasing radius represent $X_{\mathrm{CRp}}(r)$ while the declining curves show $X_{B}(r)$ for the particular choice of a magnetic field being frozen into the flow and isotropized, i.e. $\alpha_{B}=0.7$ (Tribble 1993).

of $X_{\mathrm{CRp}}(r)$ and the ratio of magnetic-to-thermal energy density $X_{B}(r)=\varepsilon_{B}(r) / \varepsilon_{\mathrm{th}}(r)$ for particular model parameters $\alpha_{\mathrm{p}}$, $\alpha_{B}$, and $B_{0}$. Whereas $\alpha_{\mathrm{p}}$ and $B_{0}$ impact mostly on the normalization of both scaling parameters $X_{\mathrm{CRp}}(r)$ and $X_{B}(r)$, the choice of $\alpha_{B}$ governs the relative curvature of these functions: $X_{B}(r) \propto n_{\mathrm{e}}(r)^{2 \alpha_{B}-1}$ is curved in a convex fashion for $\alpha_{B}>0.5$ and exhibits concave curvature for $\alpha_{B}<0.5$ assuming the cluster to be isothermal which is a valid approximation for Coma. While there are combinations of parameters for which $X_{\mathrm{CRp}}(r)$ becomes larger than unity and thus question the hadronic scenario (Brunetti 2002), only small variations in parameter space yield plausible values for $X_{\mathrm{CRp}}(r)$ (compare Fig. 6).

In order to quantify these considerations we perform a parameter study to exclude regions of parameter space spanned by $\alpha_{\mathrm{p}}, \alpha_{B}$, and $B_{0}$ where the hadronic scenario is challenged to account for the radio halo in Coma. Figure 7 shows the resulting contour lines of $X_{\mathrm{CRp}}\left(r \leq 1 h_{70}^{-1} \mathrm{Mpc}\right)=1$ and $X_{\mathrm{CRp}}\left(r \leq 1 h_{70}^{-1} \mathrm{Mpc}\right)=0.1$ in this parameter space. The gradient of $X_{\mathrm{CRp}}\left(r \leq 1 h_{70}^{-1} \mathrm{Mpc}\right)$ points towards the lower right corner in Fig. 7 and thus leaves the upper left region of parameter space where the hadronic scenario is able to account for the observed radio halo depending on the specific choice of $\alpha_{B}$. Since $X_{B}\left(r \leq 1 h_{70}^{-1} \mathrm{Mpc}\right)<0.1$ for the entire region of parameter space investigated here there are no further constraints imposed on the hadronic scenario.

Choosing the energy density of the magnetic field to decline like the thermal energy density, i.e. $\alpha_{B}=0.5$, requires $X_{\mathrm{CRp}}(r)$ to increase by a factor of less than two orders of magnitude from the center to the outer parts of the cluster in order to reproduce the observed radio halo of Coma. This factor, however, is reduced for smaller values of $\alpha_{B}$. It is further reduced due to the non-spherical morphology of Coma, as explained in the following. The X-ray emissivity and the radio emissivity 


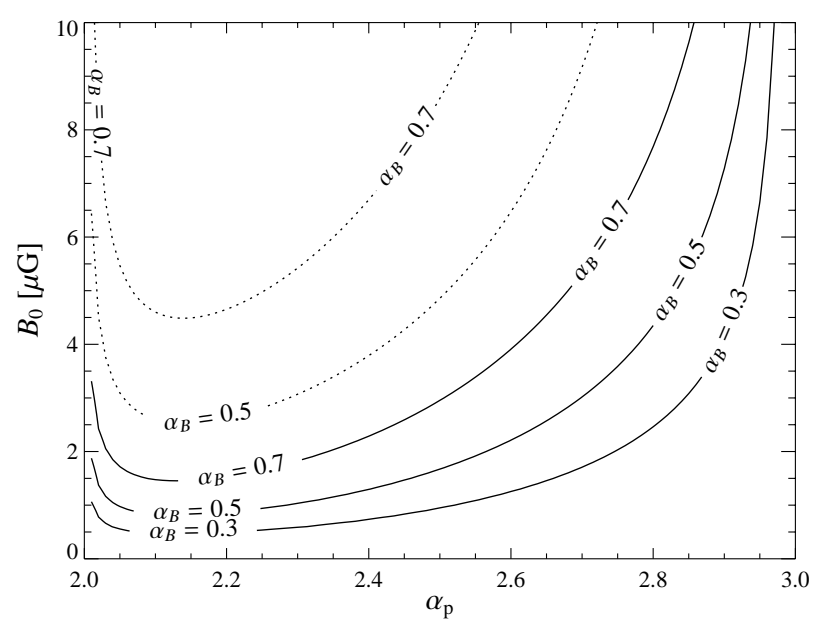

Fig. 7. Parameter study on the ability of hadronically originating CRe to generate the radio halo of Coma. Shown are contour lines of $X_{\mathrm{CRp}}\left(r \leq 1 h_{70}^{-1} \mathrm{Mpc}\right)=1$ (solid) and $X_{\mathrm{CRp}}\left(r \leq 1 h_{70}^{-1} \mathrm{Mpc}\right)=0.1$ (dotted) in parameter space spanned by $\alpha_{\mathrm{p}}$ and $B_{0}$ for three choices of magnetic field morphology characterized by $\alpha_{B}$. The contour line of $X_{\mathrm{CRp}}=0.1$ for $\alpha_{B}=0.3$ has been omitted since it almost coincides with the contour of $X_{\mathrm{CRp}}=1$ for $\alpha_{B}=0.7$. The lower right corner represents the region in parameter space, where the hadronic scenario faces challenges for explaining the observed radio halo of Coma.

resulting from hadronic CRp interactions differ in their scaling with the electron density according to

$\Lambda_{\mathrm{X}}(r) \propto n_{\mathrm{e}}(r)^{2} \quad$ and

$j_{v}(r) \propto X_{\mathrm{CRp}}(r) n_{\mathrm{e}}(r)^{2+\alpha_{B}\left(1+\alpha_{\mathrm{p}} / 2\right)} \sim X_{\mathrm{CRp}}(r) n_{\mathrm{e}}(r)^{3 \ldots 4}$

within the framework set by our model and depending on the particular choice of $\alpha_{B}$ and $\alpha_{\mathrm{p}}$. Thus, any anisotropy like the Coma X-ray and radio bridge yields biased azimuthal averages when comparing observational to theoretical radio surface brightness profiles where the latter uses density profiles obtained by deprojecting X-ray profiles. Remarkably, this discrepancy is largest for large values of $\alpha_{B}$ and $\alpha_{\mathrm{p}}$ for which we infer the tightest limits on the hadronic scenario (cf. Fig. 7) and thus softens these limits. This results in biased profiles of $X_{\mathrm{CRp}}(r)$ which increase too strongly towards larger radii (cf. also Dolag \& Enßlin 2000). Pursuing an approach of averaging only along the line of sight could attenuate the bias (Govoni et al. 2001).

An increase of $X_{\mathrm{CRp}}(r)$ towards the cluster's periphery is indeed observed in cosmological structure formation simulations due to adiabatic compression inside the cluster which increases the thermal pressure at a higher rate than the CRp pressure (Miniati et al. 2001a,b). Bearing in mind that the CRpto-thermal pressure ratio of Miniati et al. $(2001 \mathrm{a}, \mathrm{b})$ is obtained from volume averages and the energy density stored in magnetic fields declines shallower in comparison to the thermal energy density we conclude that our results arising the parameter study may be well in agreement with these simulations.

\subsubsection{The spectrum of the Coma radio halo}

One might object that the CRp spectral index should be determined better owing to radio observations than the range of

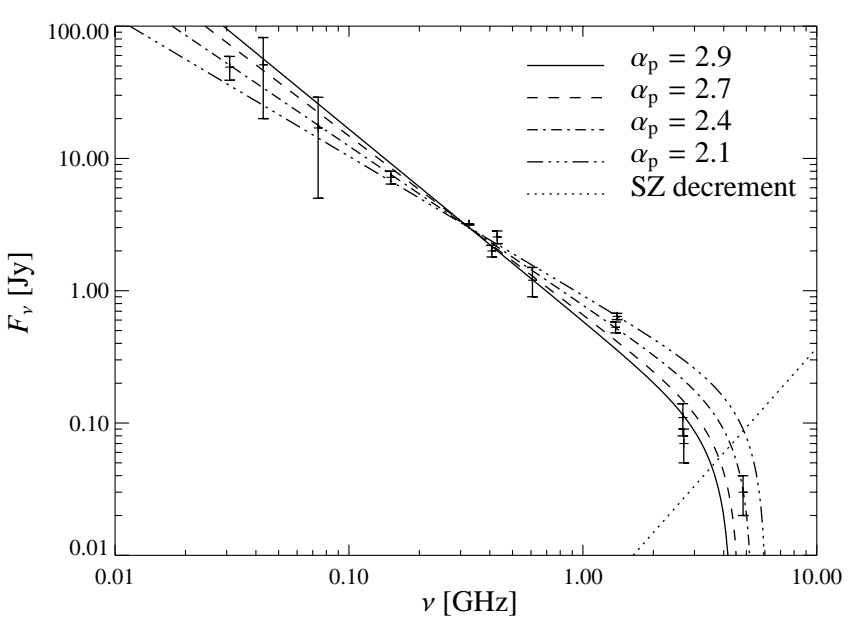

Fig. 8. Observed radio halo fluxes of the Coma cluster as compiled by Thierbach et al. (2003). Shown are synchrotron power-law spectra for different spectral indices $\alpha_{v}=\alpha_{\mathrm{p}} / 2$. The spectra are modified at higher frequencies by means of the SZ effect while the SZ decrement (a negative flux at the Rayleigh-Jeans part of the Planck spectrum) is also shown (dotted). The SZ flux is derived from the cluster volume up to the assumed position of the accretion shock.

$\alpha_{\mathrm{p}}=(2,3)$ being considered in the previous parameter study (Sect. 3.5.1). The following line of argumentation shows, that this, on the contrary, is not the case. First, there is an ambiguity of relating the CRp spectral index $\alpha_{\mathrm{p}}$ to the induced synchrotron spectral index $\alpha_{v}$ which is either $\alpha_{v}=\alpha_{\mathrm{p}} / 2$ (Dermer's model) or $\alpha_{v}=\left(2 \alpha_{\mathrm{p}}-1\right) / 3$ (fireball model). When comparing multifrequency observations of diffuse radio emission of the ICM which extends to several GHz the Sunyaev-Zel'dovich (SZ) distortion of the spectrum has to be taken care of. At these frequencies of the Rayleigh-Jeans part of the Planck spectrum the SZ effect amounts to a decrement which introduces a cutoff in the radio spectrum as can be seen in Fig. 8. Following Enßlin (2002) the SZ luminosity reads for Coma in the Rayleigh-Jeans part

$F_{\mathrm{SZ}}^{\mathrm{Coma}}=-4.1 \times 10^{-3} v_{\mathrm{GHz}}^{2} h_{70}^{-1 / 2} \mathrm{Jy}$,

where $v_{\mathrm{GHz}}=v / \mathrm{GHz} .^{3}$ However, the SZ amplitude is uncertain within a factor of 2 which stems mostly from density profiles being inferred from X-ray observations and extrapolated to $R_{\text {shock}}$. Furthermore, the multifrequency dataset as compiled by Thierbach et al. (2003) is inhomogeneous because the solid angle over which the observed radio fluxes have been integrated may vary among these observations. Finally, the quoted uncertainties may underestimate the systematic uncertainties which e.g. result from incomplete accounting for point source subtraction.

\section{Detectability of $\gamma$-rays by satellite missions and Čerenkov telescopes}

Based on the previous results we discuss the detectability of IC emission by secondary CRe and pion decay induced $\gamma$-ray

\footnotetext{
${ }^{3}$ Here we corrected for a missing factor of 2 in Eq. (4) in Enßlin (2002) and changed the slope of the $\beta$-profile to $\beta=0.75$ (Briel et al. 1992).
} 
emission by current and future satellite missions as well as operating and future Čerenkov telescopes.

\subsection{Detectability of pion decay induced $\gamma$-ray and IC emission by secondary $\mathrm{CRe}$ with INTEGRAL}

The imager IBIS which is the Imager on Board the "INTErnational Gamma-Ray Astrophysics Laboratory" (INTEGRAL) ${ }^{4}$ Satellite covers an energy range from $15 \mathrm{keV}$ up to $10 \mathrm{MeV}$ and is capable of high resolution imaging $\left(12^{\prime}\right.$ FWHM) and source identification. Its spectral sensitivity reaches down to $5 \times 10^{-8} \gamma \mathrm{s} \mathrm{s}^{-1} \mathrm{~cm}^{-2} \mathrm{keV}^{-1}$ ( $3 \sigma$ in $10^{6} \mathrm{~s}$, $\Delta E=E / 2)$ to the continuum at $10 \mathrm{MeV}$. However, this is most probably not sufficient in order to detect the pion decay induced $\gamma$-rays of a particular cluster (compare Fig. 3). Assuming a CRp spectral index of $\alpha_{\mathrm{p}}=2.3$ and taking the results of Table 5 we expect an IC emission of hadronically originating $\mathrm{CRe}$ in the Perseus cluster of

$\frac{\mathrm{d} \mathcal{F}}{\mathrm{d} E}(20 \mathrm{keV})=\mathcal{F}_{\mathrm{IC}} 10^{-7} \gamma \mathrm{cm}^{-2} \mathrm{~s}^{-1} \mathrm{keV}^{-1}$,

with $\mathcal{F}_{\mathrm{IC}}=2.9,1.5$, and 0.8 for $B_{0}=5 \mu \mathrm{G}, 10 \mu \mathrm{G}$, and $20 \mu \mathrm{G}$. By comparing to the spectral sensitivity of $2 \times$ $10^{-6} \gamma \mathrm{s}^{-1} \mathrm{~cm}^{-2} \mathrm{keV}^{-1}$ to the continuum at $20 \mathrm{keV}$ for an observation time of $10^{6} \mathrm{~s}(3 \sigma$ detection) there is only a minor chance to detect IC emission of CRe. However, for steeper spectral indices or a strongly inhomogeneously magnetized environment, IC fluxes can be enhanced at the expense of synchrotron emission according to Enßlin et al. (1999).

\subsection{Possibility of pion decay induced $\gamma$-ray detection by GLAST}

The "Large Area Telescope" (LAT) onboard the "Gammaray Large Area Space Telescope" (GLAST) ${ }^{5}$ scheduled to be launched in 2006 has an angular resolution smaller than $3.5^{\circ}$ at $100 \mathrm{MeV}$ while covering an energy range of $20 \mathrm{MeV}$ up to $300 \mathrm{GeV}$ with an energy resolution smaller than $10 \%$. Assuming a photon spectral index of $\alpha_{\gamma}=2$ for the $\gamma$-ray background the point source sensitivity at high galactic latitude in an one year all-sky survey is better than $6 \times 10^{-9} \mathrm{~cm}^{-2} \mathrm{~s}^{-1}$ for energies integrated above $100 \mathrm{MeV}$. Assuming the radiomini halo in the Perseus cluster mainly to originate from secondary electrons emitting synchrotron radiation then we expect the CRp scaling parameter to be typically one order of magnitude below the upper limits obtained by comparing to EGRET data. This immediately would imply a good possibility to detect pion decay induced $\gamma$-ray emission by GLAST preferentially in nearby cooling flow clusters like Perseus and Virgo. Specifically for our secondary model of the radio minihalo of Perseus, while assuming $\alpha_{\mathrm{p}}=2.3$ in the CRp isobaric model we expect an integrated $\gamma$-ray flux above $100 \mathrm{MeV}$ from Perseus of $\mathcal{F}_{\gamma}(>100 \mathrm{MeV}) /\left(\gamma \mathrm{cm}^{-2} \mathrm{~s}^{-1}\right)=1.3 \times 10^{-8}$, $7.4 \times 10^{-9}$, and $5.6 \times 10^{-9}$ for $B_{0}=5 \mu \mathrm{G}, 10 \mu \mathrm{G}$, and $20 \mu \mathrm{G}$.

\footnotetext{
${ }^{4}$ http://astro.esa.int/Integral/

5 http://glast.gsfc.nasa.gov/science/
}

The expected $\gamma$-ray flux is ever higher when including lower energetic photons.

\subsection{Expected $\gamma$-ray flux for Čerenkov telescopes}

In the near future there will be different Čerenkov telescope experiments operating with several telescopes simultaneously and therefore allowing stereoscopic observations. On the southern hemisphere there are the "Collaboration between Australia and Nippon for a Gamma Ray Observatory in the Outback" (CANGAROO) ${ }^{6}$ in Australia and the "High Energy Stereoscopic System" (HESS) ${ }^{7}$ in Namibia. On the northern hemisphere there will be the "Very Energetic Radiation Imaging Telescope Array System" (VERITAS) ${ }^{8}$ in Arizona. All these telescopes have comparable lower energy thresholds of $E_{\mathrm{thr}}=100 \mathrm{GeV}$ and provide flux sensitivities better than $\mathcal{F}_{\gamma, \text { exp }}(E>100 \mathrm{GeV})=10^{-12} \gamma \mathrm{cm}^{-2} \mathrm{~s}^{-1}$. On the northern hemisphere there will also be the "Major Atmospheric Gammaray Imaging Čerenkov detector" (MAGIC) ${ }^{9}$ on the Canary Islands observing with a single dish telescope of $234 \mathrm{~m}^{2}$ providing an even lower energy threshold of $E_{\min }=30 \mathrm{GeV}$.

Following the formalism described in Sect. 3.3.2 and comparing the resulting $\gamma$-ray flux $\mathcal{F}_{\gamma}\left(E>E_{\text {thr }}\right)$ to expected flux sensitivities of Čerenkov telescopes $\mathcal{F}_{\gamma, \exp }\left(E>E_{\text {thr }}\right)$, we obtain possible upper limits on the CRp scaling parameter $X_{\mathrm{CRp}}$ for an integrated volume out to a radial distance of $3 h_{70}^{-1} \mathrm{Mpc}$. Table 6 shows constraints for $X_{\mathrm{CRp}}$ using the isobaric and the adiabatic model of CRp described in Sect. 3.2. By comparing these limits to those obtained by analyzing synchrotron emission in the Perseus and Coma cluster (see Table 5) and assuming a substantial contribution of hadronically originating CRe to these radio halos there is a realistic chance to detect extragalactic pion decay induced $\gamma$-ray emission in clusters like Perseus (A 426), Virgo, Ophiuchus, and Coma (A 1656).

\section{Conclusion}

We investigated hadronic CRp-p interactions in the ICM of clusters and simulated the resulting emission mechanisms in radio, X-rays, and $\gamma$-rays assuming spherical symmetry. By applying this technique to a sample of prominent clusters of galaxies including cooling flow clusters we succeeded in constraining the population of CRp. Especially cooling flow regions are perfectly suited for constraining non-thermal ICM components due to their high gas density and magnetic field strength.

For the first time we developed an analytic formalism to describe the $\pi^{0}$-decay induced $\gamma$-ray spectrum self-consistently for a given differential number density distribution of the CRp population being described by a power-law in momentum $p_{\mathrm{p}}$ and parametrized by the spectral index $\alpha_{\mathrm{p}}$. Assuming a constant scaling between kinetic CRp energy density and thermal energy density of the ICM we derived an analytic $\mathcal{F}_{\gamma}-F_{\mathrm{X}}$

\footnotetext{
${ }^{6}$ http://www .physics.adelaide.edu.au/astrophysics/

7 http://www.mpi-hd.mpg.de/hfm/HESS/HESS.html

8 http://veritas.sao.arizona.edu/

${ }^{9}$ http://hegra1.mppmu.mpg.de/MAGICWeb/
} 
Table 6. Expected limits on the CRp scaling parameter $X_{\mathrm{CRp}}$ by comparing the integrated pion decay induced $\gamma$-ray flux above $100 \mathrm{GeV}$ to sensitivity limits of Čerenkov telescopes of $\mathcal{F}_{\gamma \text {,exp }}\left(E>E_{\mathrm{thr}}\right)=10^{-12} \gamma \mathrm{cm}^{-2} \mathrm{~s}^{-1}\left(E_{\mathrm{thr}} / 100 \mathrm{GeV}\right)^{1-\alpha_{\gamma}}$ assuming a $\gamma$-ray spectral index in Dermer's model $\alpha_{\gamma}=\alpha_{\mathrm{p}}$. Note that limits on $X_{\mathrm{CRp}}$ roughly $\lessgtr 0.01$ for $\alpha_{\mathrm{p}}=2.3$ in the isobaric model provide good chances to detect $\gamma$-rays in these particular clusters with new generation Čerenkov telescopes.

\begin{tabular}{|c|c|c|c|c|c|c|c|c|}
\hline \multirow[b]{2}{*}{ Cluster } & \multicolumn{4}{|c|}{$X_{\text {CRp }}^{\text {isobaric }}$} & \multicolumn{4}{|c|}{$X_{\mathrm{CRp}}^{\text {adiabatic }}$} \\
\hline & $\alpha_{\mathrm{p}}=2.1$ & $\alpha_{\mathrm{p}}=2.3$ & $\alpha_{\mathrm{p}}=2.5$ & $\alpha_{\mathrm{p}}=2.7$ & $\alpha_{\mathrm{p}}=2.1$ & $\alpha_{\mathrm{p}}=2.3$ & $\alpha_{\mathrm{p}}=2.5$ & $\alpha_{\mathrm{p}}=2.7$ \\
\hline A85 & $3.7 \times 10^{-2}$ & $6.5 \times 10^{-2}$ & $2.2 \times 10^{-1}$ & 1.0 & $2.7 \times 10^{-2}$ & $4.7 \times 10^{-2}$ & $1.5 \times 10^{-1}$ & $7.1 \times 10^{-1}$ \\
\hline A426 (Perseus) & $2.5 \times 10^{-3}$ & $4.5 \times 10^{-3}$ & $1.5 \times 10^{-2}$ & $7.0 \times 10^{-2}$ & $2.1 \times 10^{-3}$ & $3.6 \times 10^{-3}$ & $1.2 \times 10^{-2}$ & $5.5 \times 10^{-2}$ \\
\hline A2199 ....... & $4.4 \times 10^{-2}$ & $7.7 \times 10^{-2}$ & $2.6 \times 10^{-1}$ & 1.2 & $4.1 \times 10^{-2}$ & $7.2 \times 10^{-2}$ & $2.4 \times 10^{-1}$ & 1.1 \\
\hline A3526 (Centaurus) & $1.9 \times 10^{-2}$ & $3.4 \times 10^{-2}$ & $1.1 \times 10^{-1}$ & $5.3 \times 10^{-1}$ & $1.8 \times 10^{-2}$ & $3.2 \times 10^{-2}$ & $1.1 \times 10^{-1}$ & $5.0 \times 10^{-1}$ \\
\hline Ophiuchus ........ & $4.0 \times 10^{-3}$ & $7.0 \times 10^{-3}$ & $2.3 \times 10^{-2}$ & $1.1 \times 10^{-1}$ & & & & \\
\hline Triangulum Australis & $1.6 \times 10^{-2}$ & $2.8 \times 10^{-2}$ & $9.3 \times 10^{-2}$ & $4.4 \times 10^{-1}$ & & & & \\
\hline Virgo ............. & $4.1 \times 10^{-3}$ & $7.3 \times 10^{-3}$ & $2.4 \times 10^{-2}$ & $1.1 \times 10^{-1}$ & $3.8 \times 10^{-3}$ & $6.7 \times 10^{-3}$ & $2.2 \times 10^{-2}$ & $1.0 \times 10^{-1}$ \\
\hline A1656 (Coma) ..... & $7.9 \times 10^{-3}$ & $1.4 \times 10^{-2}$ & $4.6 \times 10^{-2}$ & $2.2 \times 10^{-1}$ & & & & \\
\hline A $2256 \ldots \ldots \ldots \ldots$ & $4.9 \times 10^{-2}$ & $8.6 \times 10^{-2}$ & $2.9 \times 10^{-1}$ & 1.4 & & & & \\
\hline A $2319 \ldots \ldots \ldots$ & $1.5 \times 10^{-2}$ & $2.6 \times 10^{-2}$ & $8.9 \times 10^{-2}$ & $4.2 \times 10^{-1}$ & & & & \\
\hline A3571 .......... & $1.9 \times 10^{-2}$ & $3.4 \times 10^{-2}$ & $1.1 \times 10^{-1}$ & $5.3 \times 10^{-1}$ & & & & \\
\hline
\end{tabular}

scaling relation which only applies accurately for isothermal clusters. Given the bolometric X-ray luminosity of a particular cluster this formula estimates the expected $\gamma$-ray flux $\mathcal{F}_{\gamma}$ owing to inelastic cosmic ray ion collisions. From the literature we collected electron density and temperature profiles of seven cooling flow clusters and four non-cooling flow clusters using the $\mathcal{F}_{\gamma}-F_{\mathrm{X}}$ scaling relation to obtain observationally promising candidates. We furthermore present formulae describing the synchrotron and inverse Compton emission of hadronically originating secondary electrons assuming an isotropic distribution of magnetic fields following a smooth profile.

In order to apply this method to our sample of clusters of galaxies we introduced three specific models for the spatial distribution of CRp within cooling flow cluster. In our first two scenarios we characterized the kinetic CRp energy density $\varepsilon_{\mathrm{CRp}}(\boldsymbol{r})$ to be a constant fraction of the thermal energy density $\varepsilon_{\mathrm{th}}(\boldsymbol{r})$ of the ICM parametrized by $X_{\mathrm{CRp}}$. The CRp isobaric model assumes the average pressure of CRp not to change during the formation of the cooling flow while the adiabatic model hypothesizes this proportionality prior to transition because the CRp experience adiabatic compression during the relaxation phase. In our third scenario we modeled the resulting distribution of CRp diffusion from a central source. By modeling the particular $\gamma$-ray emission of our cluster sample and comparing to EGRET upper limits we obtained upper bounds on the CRp scaling parameter $X_{\mathrm{CRp}}=\varepsilon_{\mathrm{CRp}}(\boldsymbol{r}) / \varepsilon_{\mathrm{th}}(\boldsymbol{r})$. For Perseus and Virgo we infer the strongest upper limits which lie in the range $X_{\mathrm{CRp}} \in[0.08,0.18]$ for different choices of the CRp spectral index $\alpha_{\mathrm{p}} \in[2.1,2.7]$.

Furthermore, the radio emission due to hadronically produced secondary electrons emitting synchrotron radiation was calculated and resulting radio brightness profiles were compared to measured data of the radio-mini halo of Perseus as well as the radio halo of Coma. In the case of Coma our CRp profiles characterized by a flat CRp scaling parameter $X_{\mathrm{CRp}}$ are not able to reproduce the observed radio profiles particularly in the peripheral regions of the cluster. In the following we adjusted the radial behavior of $X_{\mathrm{CRp}}(r)$ such that the synchrotron emission resulting from hadronic $\mathrm{CRe}$ is able to account for the observed radio surface brightness profile and thus allowing for an additional degree of freedom. The resulting increase of $X_{\mathrm{CRp}}(r)$ for larger radii could be due to adiabatic compression which increases the thermal energy density at a higher rate than the CRp energy density. Even more important, the aspherical Coma cluster morphology reduces the required radial increase in $X_{\mathrm{CRp}}(r)$. By exploring the accessible parameter space spanned by parameters describing the magnetic field and the spectral index of the CRp population we identify regions where the hadronic scenario is able to reproduce the observed radio profiles preferentially for an energy density of the magnetic field which declines shallower than the thermal energy density. We conclude that the secondary model for radio halos is still viable.

In the case of the Perseus mini-radio halo, we conclude upper limits on $X_{\mathrm{CRp}}$ which are ranging for the isobaric model of CRp within the interval $X_{\mathrm{CRp}} \in[0.01,0.1]$ for conservative combinations of values of the magnetic field $B$ and the CRp spectral index $\alpha_{\mathrm{p}}$ while upper limits for the CRp adiabatic model are typically half an order of magnitude below. By comparing calculated radio brightness profiles to measured data of the radio-mini halo in Perseus, we found excellent morphological agreement between the CRp isobaric model and the radio data especially for the choice of $B_{0}=10 \mu \mathrm{G}, \alpha_{B}=0.5$, and $\alpha_{\mathrm{p}}=2.3$. In the course of this paper we argued that this specific choice of parameters for the magnetic fields in cooling flow clusters is also preferred by experiments like Faraday rotation measurements and cosmological cluster simulations including magnetic fields. A discussion of different acceleration mechanisms of CRp such as structure formation shocks, supernovae remnants, and injection by active radio galaxies supports also a value of $\alpha_{\mathrm{p}}$ close to the inferred one. Because of the required moderate CRp energy density we propose synchrotron radiation by non-thermal secondary electrons from hadronic interactions as a likely explanation of radio mini-halos. In order to 
scrutinize this model we provide predictions of $\gamma$-ray fluxes for Čerenkov telescopes as well as the INTEGRAL and GLAST satellites.

Finally, we analyzed the possibility of detecting such pion decay induced $\gamma$-ray and IC emission by current and future satellite missions as well as new generation Čerenkov telescopes. Depending on the CRp spectral index, the fragmentation of the spatial distribution of the magnetic field as well as its field strength, it will be difficult for INTEGRAL to detect the IC emission of the hadronically originating secondary CRe while GLAST has the potentiality to detect the distinct signature of the pion decay induced $\gamma$-ray emission preferentially in nearby cooling flow clusters. By investigating the opportunity of detecting extragalactic $\gamma$-rays by Čerenkov telescopes we argued in favor of four candidate clusters (Perseus (A 426), Virgo, Ophiuchus, and Coma (A 1656)) which are especially suited to detect hadronically originating $\gamma$-ray emission.

Acknowledgements. In particular we are indebted to Francesco Miniati for fruitful discussions and providing numerical $\gamma$-ray spectra. We also wish to thank Matthias Bartelmann, Björn Malte Schäfer and an anonymous referee for carefully reading the manuscript and their numerous constructive remarks. Furthermore, we acknowledge useful discussions with Eugene Churazov and Sebastian Heinz. This work was performed within the framework of the European Community Research and Training Network The Physics of the Intergalactic Medium.

\section{Appendix A: Deprojection of X-ray surface brightness profiles represented by double $\beta$-models}

Owing to the enhanced electron density in the central region the $\mathrm{X}$-ray surface brightness profile $S_{\mathrm{X}}\left(r_{\perp}\right)$ in cooling flow cluster can be represented by double $\beta$ models,

$S_{\mathrm{X}}\left(r_{\perp}\right)=\sum_{i=1}^{2} S_{i}\left[1+\left(\frac{r_{\perp}}{r_{\mathrm{c}_{i}}}\right)^{2}\right]^{-3 \beta_{i}+1 / 2}$,

where the X-ray surface brightness profile is a line of sight projection of the squared electron density and the cooling function relative to the squared electron density $\tilde{\Lambda}_{\mathrm{X}}\left(T_{\mathrm{e}}\right)$,

$$
\begin{aligned}
S_{\mathrm{X}}\left(r_{\perp}\right) & =\int_{-\infty}^{\infty} \mathrm{d} z n_{\mathrm{e}}^{2}\left(\sqrt{r_{\perp}^{2}+z^{2}}\right) \tilde{\Lambda}_{\mathrm{X}}\left[T_{\mathrm{e}}\left(\sqrt{r_{\perp}^{2}+z^{2}}\right)\right] \\
& =2 \int_{r_{\perp}}^{\infty} \mathrm{d} r \frac{r n_{\mathrm{e}}^{2}(r) \tilde{\Lambda}_{\mathrm{X}}\left[T_{\mathrm{e}}(r)\right]}{\sqrt{r^{2}-r_{\perp}^{2}}} .
\end{aligned}
$$

Thus the electron density $n_{\mathrm{e}}(r)$ can be derived from $S_{\mathrm{X}}\left(r_{\perp}\right)$ by inverting the Abel equation

$$
\begin{aligned}
n_{\mathrm{e}}^{2}(r) \tilde{\Lambda}_{\mathrm{X}}\left[T_{\mathrm{e}}(r)\right] & =-\frac{1}{\pi r} \frac{\mathrm{d}}{\mathrm{d} r} \int_{r}^{\infty} \mathrm{d} y \frac{y S_{\mathrm{X}}(y)}{\sqrt{y^{2}-r^{2}}} \\
& =-\frac{1}{\pi} \int_{r}^{\infty} \mathrm{d} y \frac{S_{\mathrm{X}}^{\prime}(y)}{\sqrt{y^{2}-r^{2}}},
\end{aligned}
$$

where the prime denotes the derivative. For the second equation we used that $n_{\mathrm{e}}(r)$ is bounded for $r \rightarrow \infty$. Using Eq. (A.1) this equation can be solved analytically yielding

$$
n_{\mathrm{e}}^{2}(r)=\frac{1}{\tilde{\Lambda}_{\mathrm{X}}\left[T_{\mathrm{e}}(r)\right]} \sum_{i=1}^{2} \frac{S_{i}}{2 \pi r_{\mathrm{c}_{i}}} \frac{6 \beta_{i}-1}{\left(1+r^{2} / r_{\mathrm{c}_{i}}^{2}\right)^{3 \beta_{i}}} \mathcal{B}\left(\frac{1}{2}, 3 \beta_{i}\right),
$$

where $\mathcal{B}(a, b)$ denotes the beta-function (Abramowitz \& Stegun $1965)$. Provided the central density $n_{\mathrm{e}}(0)$ is known and assuming furthermore the special case of equality of the two $\beta$ parameter, $\beta_{1}=\beta_{2}$, we arrive at the following compact formula for the electron density profile $n_{\mathrm{e}}(r)$

$$
\begin{aligned}
& n_{\mathrm{e}}(r)=\left[\frac{\tilde{\Lambda}_{\mathrm{X}}\left[T_{\mathrm{e}}(0)\right]}{\tilde{\Lambda}_{\mathrm{X}}\left[T_{\mathrm{e}}(r)\right]} \times \sum_{i=1}^{2} n_{i}^{2}\left(1+\frac{r^{2}}{r_{\mathrm{c}_{i}}^{2}}\right)^{-3 \beta}\right]^{1 / 2}, \\
& n_{i}=n_{\mathrm{e}}(0)\left(\sum_{j=1}^{2} \frac{S_{j} r_{\mathrm{c}_{i}}}{S_{i} r_{\mathrm{c}_{j}}}\right)^{-1 / 2} .
\end{aligned}
$$

Generalizing to $n$-fold $\beta$-profiles can be obtained by means of induction.

\section{References}

Abramowitz, M., \& Stegun, I. A. 1965, Handbook of mathematical functions (Dover Books on Advanced Mathematics, New York: Dover)

Allen, S. W., Schmidt, R. W., \& Fabian, A. C. 2001, MNRAS, 328, L37

Arnaud, M., Aghanim, N., Gastaud, R., et al. 2001, A\&A, 365, L67

Badhwar, G. D., Golden, R. L., \& Stephens, S. A. 1977, Phys. Rev. D, 15,820

Berezinsky, V. S., Blasi, P., \& Ptuskin, V. S. 1997, ApJ, 487, 529

Bicknell, G. V., \& Begelman, M. C. 1996, ApJ, 467, 597

Blasi, P. 1999, ApJ, 525, 603

Blasi, P., \& Colafrancesco, S. 1999, Astropart. Phys., 12, 169

Briel, U. G., Henry, J. P., \& Böhringer, H. 1992, A\&A, 259, L31

Brunetti, G. 2002, in Matter and Energy in Clusters of Galaxies, ed. S. Bowyer, \& C.-Y. Hwang, ASP Conf. Ser., in preparation [astro-ph/0208074]

Brunetti, G., Feretti, L., Giovannini, G., \& Setti, G. 1999, in Diffuse Thermal and Relativistic Plasma in Galaxy Clusters, 263

Brunetti, G., Setti, G., Feretti, L., \& Giovannini, G. 2001, MNRAS, 320,365

Carilli, C. L., \& Taylor, G. B. 2002, ARA\&A, 40, 319

Churazov, E., Brüggen, M., Kaiser, C. R., Böhringer, H., \& Forman, W. 2001, ApJ, 554, 261

Churazov, E., Forman, W., Jones, C., \& Böhringer, H. 2003, ApJ, 590, 225

Colafrancesco, S., \& Blasi, P. 1998, Astropart. Phys., 9, 227

David, L. P., Slyz, A., Jones, C., et al. 1993, ApJ, 412, 479

Deiss, B. M., Reich, W., Lesch, H., \& Wielebinski, R. 1997, A\&A, 321,55

Dennison, B. 1980, ApJ, 239, L93

Dermer, C. D. 1986a, ApJ, 307, 47

Dermer, C. D. 1986b, A\&A, 157, 223

Dolag, K., Bartelmann, M., \& Lesch, H. 1999, A\&A, 348, 351

Dolag, K., \& Enßlin, T. A. 2000, A\&A, 362, 151

Dolag, K., Schindler, S., Govoni, F., \& Feretti, L. 2001, A\&A, 378, 777 
Ebeling, H., Edge, A. C., Böhringer, H., et al. 1998, MNRAS, 301, 881

Enßlin, T. A. 2002, A\&A, 396, L17

Enßlin, T. A. 2003, A\&A, 399, 409

Enßlin, T. A., Biermann, P. L., Klein, U., \& Kohle, S. 1998a, A\&A, 332, 395

Enßlin, T. A., Biermann, P. L., Kronberg, P. P., \& Wu, X.-P. 1997, ApJ, 477,560

Enßlin, T. A., \& Brüggen, M. 2002, MNRAS, 331, 1011

Enßlin, T. A., \& Gopal-Krishna 2001, A\&A, 366, 26

Enßlin, T. A., Lieu, R., \& Biermann, P. L. 1999, A\&A, 344, 409

Enßlin, T. A., \& Vogt, C. 2003, A\&A, 401, 835

Enßlin, T. A., Wang, Y., Nath, B. B., \& Biermann, P. L. 1998b, A\&A, 333, L47

Fermi, E. 1950, Prog. Theor. Phys., 5, 570

Fusco-Femiano, R., dal Fiume, D., Feretti, L., et al. 1999, ApJ, 513, L21

Giovannini, G., Feretti, L., Venturi, T., Kim, K. T., \& Kronberg, P. P. 1993, ApJ, 406, 399

Gitti, M., Brunetti, G., \& Setti, G. 2002, A\&A, 386, 456

Gould, R. J. 1972, Physica, 58, 379

Govoni, F., Enßlin, T. A., Feretti, L., \& Giovannini, G. 2001, A\&A, 369,441

Harris, D. E., Kapahi, V. K., \& Ekers, R. D. 1980, A\&AS, 39, 215

Irwin, J. A., \& Bregman, J. N. 2000, ApJ, 538, 543

Jaffe, W. J. 1977, ApJ, 212, 1

Johnstone, R. M., Allen, S. W., Fabian, A. C., \& Sanders, J. S. 2002, MNRAS, 336, 299

Lahav, O., Fabian, A. C., Edge, A. C., \& Putney, A. 1989, MNRAS, 238,881

Lima Neto, G. B., Pislar, V., \& Bagchi, J. 2001, A\&A, 368, 440

Mannheim, K., \& Schlickeiser, R. 1994, A\&A, 286, 983

Matsushita, K., Belsole, E., Finoguenov, A., \& Böhringer, H. 2002, A\&A, 386, 77

McHardy, I. M., Lawrence, A., Pye, J. P., \& Pounds, K. A. 1981, MNRAS, 197, 893

Miniati, F. 2001, Comp. Phys. Comm., 141, 17

Miniati, F., Jones, T. W., Kang, H., \& Ryu, D. 2001a, ApJ, 562, 233

Miniati, F., Ryu, D., Kang, H., \& Jones, T. W. 2001b, ApJ, 559, 59

Miniati, F., Ryu, D., Kang, H., et al. 2000, ApJ, 542, 608

Mohr, J. J., Mathiesen, B., \& Evrard, A. E. 1999, ApJ, 517, 627
Moskalenko, I. V., \& Strong, A. W. 1998, ApJ, 493, 694

Nachtmann, O. 1990, Elementary particle physics: Concepts and phenomena (Springer)

Oegerle, W. R., \& Hill, J. M. 2001, AJ, 122, 2858

Ohno, H., Takizawa, M., \& Shibata, S. 2002, ApJ, 577, 658

Pedlar, A., Ghataure, H. S., Davies, R. D., et al. 1990, MNRAS, 246, 477

Quilis, V., Ibanez, J. M. A., \& Saez, D. 1998, ApJ, 502, 518

Reimer, O., Pohl, M., Sreekumar, P., \& Mattox, J. R. 2003, ApJ, 588, 155

Rephaeli, Y., Ulmer, M., \& Gruber, D. 1994, ApJ, 429, 554

Roettiger, K., Burns, J. O., \& Stone, J. M. 1999, ApJ, 518, 603

Röttgering, H. J. A., Wieringa, M. H., Hunstead, R. W., \& Ekers, R. D. 1997, MNRAS, 290, 577

Rybicki, G. B., \& Lightman, A. P. 1979, Radiative processes in astrophysics (New York: Wiley-Interscience)

Sarazin, C. L. 1999, ApJ, 520, 529

Sarazin, C. L. 2002, in ASSL Vol. 272: Merging Processes in Galaxy Clusters, 1-38

Schlickeiser, R. 2002, Cosmic ray astrophysics (Springer)

Schlickeiser, R., Sievers, A., \& Thiemann, H. 1987, A\&A, 182, 21

Sijbring, L. G. 1993, Ph.D. Thesis, Groningen University

Stecker, F. W. 1970, Ap\&SS, 6, 377

Stephens, S. A., \& Badhwar, G. D. 1981, Ap\&SS, 76, 213

Struble, M. F., \& Rood, H. J. 1999, ApJS, 125, 35

Sun, M., Murray, S. S., Markevitch, M., \& Vikhlinin, A. 2002, ApJ, 565,867

Takizawa, M., \& Naito, T. 2000, ApJ, 535, 586

Thierbach, M., Klein, U., \& Wielebinski, R. 2003, A\&A, 397, 53

Tribble, P. C. 1993, MNRAS, 263, 31

Valtaoja, E. 1984, A\&A, 135, 141

Vestrand, W. T. 1982, AJ, 87, 1266

Voigt, L. M., Schmidt, R. W., Fabian, A. C., Allen, S. W., \& Johnstone, R. M. 2002, MNRAS, 335, L7

Völk, H. J., Aharonian, F. A., \& Breitschwerdt, D. 1996, Space Sci. Rev., 75, 279

White, D. A. 2000, MNRAS, 312, 663

Wu, K. K. S., Fabian, A. C., \& Nulsen, P. E. J. 2000, MNRAS, 318, 889

Young, A. J., Wilson, A. S., \& Mundell, C. G. 2002, ApJ, 579, 560 